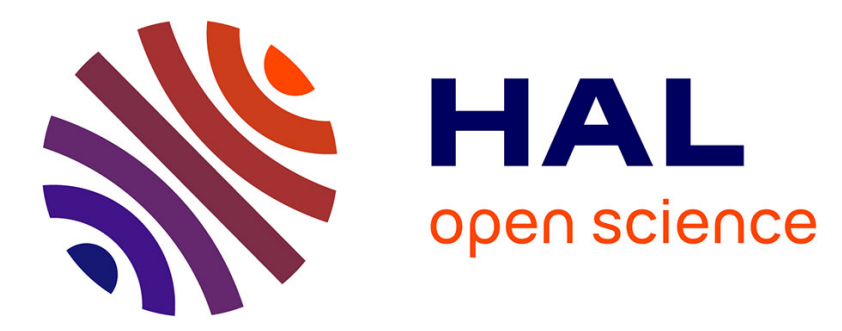

\title{
Magnetic field induced structural changes in magnetite observed by resonant x-ray diffraction and Mössbauer spectroscopy
}

T Kolodziej, I Bialo, Wojciech Tabis, M. Zubko, J Żukrowski, K. Łątka, J.E. Lorenzo, C. Mazzoli, Z Kąkol, A Kozlowski, et al.

\section{To cite this version:}

T Kolodziej, I Bialo, Wojciech Tabis, M. Zubko, J Żukrowski, et al.. Magnetic field induced structural changes in magnetite observed by resonant x-ray diffraction and Mössbauer spectroscopy. Physical Review B, 2020, 102 (7), pp.075126. 10.1103/PhysRevB.102.075126 . hal-02922539

\section{HAL Id: hal-02922539 \\ https://hal.science/hal-02922539}

Submitted on 26 Aug 2020

HAL is a multi-disciplinary open access archive for the deposit and dissemination of scientific research documents, whether they are published or not. The documents may come from teaching and research institutions in France or abroad, or from public or private research centers.
L'archive ouverte pluridisciplinaire HAL, est destinée au dépôt et à la diffusion de documents scientifiques de niveau recherche, publiés ou non, émanant des établissements d'enseignement et de recherche français ou étrangers, des laboratoires publics ou privés. 


\title{
Magnetic field induced structural changes in magnetite observed by resonant x-ray diffraction and Mössbauer spectroscopy
}

\author{
T. Kołodziej, ${ }^{1}$ I. Biało $\odot,{ }^{2,3}$ W. Tabiś,,${ }^{2,3}$ M. Zubko $\odot,{ }^{4,5}$ J. Żukrowski $\odot,{ }^{6}$ K. Łątka $\odot,{ }^{7}$ J. E. Lorenzo, ${ }^{8}$ C. Mazzoli, ${ }^{9}{ }^{10}$ Z. Kąkol, ${ }^{2}$ \\ A. Kozłowski $\odot,{ }^{2}$ Z. Tarnawski, ${ }^{2}$ E. Wilke, ${ }^{2}$ P. Babik $\odot,{ }^{11}$ V. Chlan $\odot,{ }^{12}$ R. Řezníček, ${ }^{13}$ H. Štěpánková $\odot,{ }^{12}$ P. Novák $\odot,{ }^{14}$ \\ Y. Joly $\odot,{ }^{8}$ J. Niewolski, ${ }^{2}$ and J. M. Honig ${ }^{15}$ \\ ${ }^{1}$ SOLARIS National Synchrotron Radiation Centre, Czerwone Maki 98, 30-392 Kraków, Poland \\ ${ }^{2}$ Faculty of Physics and Applied Computer Science, AGH University of Science and Technology, Aleja Mickiewicza 30, 30-059 Kraków, Poland \\ ${ }^{3}$ Institute of Solid State Physics, TU Wien, 1040 Vienna, Austria \\ ${ }^{4}$ Faculty of Science and Technology, Institute of Materials Engineering, University of Silesia, 75 Putku Piechoty 1a, 41-500 Chorzów, Poland \\ ${ }^{5}$ Department of Physics, Faculty of Science, University of Hradec Králové, Rokitanského 62, 50003 Hradec Králové, Czech Republic \\ ${ }^{6}$ Academic Center of Materials and Nanotechnology, AGH University of Science and Technology, \\ Aleja Mickiewicza 30, 30-059 Kraków, Poland \\ ${ }^{7}$ Faculty of Physics, Astronomy and Applied Computer Science, Jagiellonian University, \\ Ulica Profesor Stanistawa Łojasiewicza 11, 30-348 Kraków, Poland \\ ${ }^{8}$ Institut Néel, CNRS and Université Joseph Fourier, BP 166, 38042 Grenoble Cedex 9, France \\ ${ }^{9}$ Brookhaven National Laboratory, 98 Rochester Street, Upton, New York 11973, USA \\ ${ }^{10}$ European Synchrotron Radiation Facility, 6 Rue Jules Horowitz, 38043 Grenoble Cedex, France \\ ${ }^{11}$ Faculty of Metals Engineering and Industrial Computer Science, AGH University of Science and Technology, \\ Aleja Mickiewicza 30, 30-059 Kraków, Poland \\ ${ }^{12}$ Faculty of Mathematics and Physics, Charles University in Prague, V Holešovičkách 2, 18000 Prague 8, Czech Republic \\ ${ }^{13}$ Faculty of Physics and Earth Sciences, University of Leipzig, Linnéstrasse 5, 04103 Leipzig, Germany \\ ${ }^{14}$ Institute of Physics of ASCR, Cukrovarnická 10, 16253 Prague 6, Czech Republic \\ ${ }^{15}$ Department of Chemistry, Purdue University, West Lafayette, Indiana 47907-2084, USA
}

(Received 22 May 2020; accepted 23 July 2020; published 18 August 2020)

\begin{abstract}
When a magnetic field is applied to a single crystal of magnetite at $124 \mathrm{~K}>T>50 \mathrm{~K}$, the monoclinic $c_{M}$ axis, which is the easy magnetization axis, switches to one of the $\langle 100\rangle$ cubic directions, nearest to the direction of the magnetic field, and the phenomenon known as an axis switching (AS) occurs. A global symmetry probe, resonant x-ray scattering, and a local probe, Mössbauer spectroscopy, are used to better understand the mechanism of axis switching. The behavior of three subsystems ordered below the Verwey transition temperature $T_{V}$, i.e., lattice distortion, an orbital, and charge orderings, was observed via resonant x-ray scattering as a function of an external magnetic field. This was preceded by calculation of selected peak intensities using the FDMNES code. The Mössbauer spectroscopy studies confirmed that the magnetic field triggers electronic rearrangements and atomic displacements. The structure observed after the process of axis switching is very similar to the one obtained after cooling below $T_{V}$ with the magnetic field applied along one of the initial $\langle 100\rangle$ cubic directions and distinct from the cooling in the absence of a magnetic field. From all the experimental observations of the phenomenon done so far, it is clear that AS starts from the fluctuations between octahedral iron orbitals that ultimately lead to the Verwey transition, but also to the higher-temperature trimeron dynamics. Therefore, further observation of the axis switching may be a key point to the understanding of a majority of strongly correlated electronic behavior in magnetite as well as in other transition metal oxides.
\end{abstract}

DOI: 10.1103/PhysRevB.102.075126

\section{INTRODUCTION}

The discontinuous Verwey transition (VT) in magnetite at $T_{V}=124 \mathrm{~K}$, visible as a drop in the resistivity by two orders of magnitude, a sudden change of lattice symmetry, and discontinuities in practically all physical properties, is still not completely resolved despite the work done during the past 100 years [1]. Being a flagship of the strongly correlated electron systems, where competing interactions are present, magnetite became a testing ground for both experimental and theoretical techniques and a full explanation of the VT, down to the most basic (microscopic) level, is of the utmost importance for the understanding of interactions in solids.

Despite the recent progress in understanding the ground state of magnetite, there is still room for further work (e.g., in the case of substituted or nonstoichiometric magnetite). Deep insight into excited states presents another challenge. Nevertheless, the anomalies at the low- $T$ specific heat [2], the anomalies in AC magnetic susceptibility at approximately 20 and $60 \mathrm{~K}[3,4]$, and its multiferroic properties [5] suggest that several interactions and several subsystems, such as the 
lattice, the charge, and its distribution (orbital order), as well as magnetic subsystem, exist and exhibit themselves in the low-temperature monoclinic $C c$ phase below $T_{V}$.

These subsystems are also visible in the vicinity of $T_{V}$. The approach of $T_{V}$ from high (or low) temperatures is not homogeneous, as revealed by recent $\mathrm{x}$-ray correlation spectroscopy [6]. In addition, although sudden changes of many physical properties occur at $T_{V}$, the charge and orbital orders persist above the transition and disappear at $130 \mathrm{~K}$ [7]. These last facts were revealed by the observation of certain reflections sensitive to these orderings: While the (001) reflection measured at the Fe $K$ edge is sensitive to the charge order, the $(001 / 2)$ reflection appears at this resonance related to the orbital order.

Even above $130 \mathrm{~K}$, in the high-temperature cubic $F d \overline{3} m$ phase, the signatures of several subsystems still exist in the form of fluctuations as revealed by the diffuse scattering experiments [8] and x-ray pair distribution function (PDF) analysis [9]. The traces in the diffuse scattering are present up to room temperature and reveal that the electronic states and atomic vibrations are closely intertwined [8,10]. The structural fluctuations, as seen by PDF analysis, are observed at even higher temperatures and disappear at the Curie temperature $T_{C}$ [9]. Since this atomic arrangement in the form of trimerons, either static as at low $T$ or dynamic, is important for exchange interactions, the continuation of this arrangement across $T_{V}$ may rationalize a very subtle change of magnetization observed at the transition.

The cubic phase may be regarded as a dynamic collection of less symmetric atomic/electronic arrangements that average to a mean cubic state. The behavior of those arrangements may be linked to the still unresolved problem of the Verwey transition of first and second order [11,12]. With such a variety of phenomena and mutually cooperating subsystems, it is interesting to see to what extent those subsystems can be manipulated by an external trigger, such as an electric field [13], light [14], or a magnetic field [15,16]. Here we exploit the phenomenon discovered by Calhoun [15], an easy magnetization axis switching (AS) caused by the magnetic field, the phenomenon closely linked with crystal structure rearrangements $[17,18]$ and visible by several bulk [18-20] and local [21] techniques. Here we use resonant X-ray scattering (RXS) and Mössbauer spectroscopy (MS) as two complementary techniques to probe the changes occurring in the system during the AS process in the temperature range below but close to $T_{V}$.

Resonant X-ray scattering is a diffraction technique combining the sensitivity to the long-range periodicity of the lattice with the spectroscopic features of the ions affected by the photons at the resonance energy. In particular, RXS at the Fe resonance edge was demonstrated to be a very powerful tool to study the spin, charge, and orbital arrangements, also in strongly correlated systems [22]. The tremendous importance of this technique was demonstrated during studies of stoichiometric magnetite, which uncovered that the temperature evolution of the electronic orders is distinct from the behavior of the lattice distortions in the vicinity of the Verwey transition [7]. The question arising is how these orders respond to the external magnetic field applied below their disordering temperature.
On the other hand, MS measures Fe nuclear energy-level hyperfine splitting. Since this splitting reflects the values relevant to the $\mathrm{Fe}$ atomic valence, the hyperfine magnetic field, and the electric field gradient, it probes the sample from a real space site and atomiclike perspective, helping to answer the natural question of what the particular atom does under changing conditions during axis switching.

The article is organized as follows. First, the low- $T$ structure and the axis switching process are described to the extent important for our further discussion. Then, in Sec. II, we provide the sample details (Sec. II A) followed by the description of the experimental setup of RXS (Sec. IIB) and MS (Sec. II C). The results are presented in Sec. III, in Sec. III A for RXS and in Sec. III B for MS, and discussed in Sec. IV. A summary and conclusions follow in Sec. V.

\section{A. Crystal lattice below $\boldsymbol{T}_{V}$}

The crystal structure of magnetite above $T_{V}$ is well known to be the cubic inverse spinel (space group $F d \overline{3} m$ ) where $\mathrm{Fe}_{3} \mathrm{O}_{4}$ is expressed as $A B_{2} \mathrm{O}_{4}$, with $\mathrm{Fe} A$ ions located in tetrahedral positions and $\mathrm{Fe} B$ ions in octahedral positions. Below $T_{V}$, the structure becomes $C c$ and the low symmetry reflects the complexity of the phenomena undergone. The basis vectors $a_{M}, b_{M}$, and $c_{M}$ of the monoclinic unit cell roughly coincide with the [1-1 0], [110], and [001] directions of the original cubic lattice. The monoclinic $c_{M}$ axis is tilted $\sim 0.20^{\circ}$ away from the vertical towards the $-a_{M}$ direction. The average displacements of atoms, as compared to cubic symmetry, are reported to be of the order of $0.1 \AA[23,24]$.

The unit $C c$ cell is four times bigger than the cubic one and consists of 224 atoms [25,26], with eight tetrahedral nonequivalent Fe positions, all of $3+$ valence and 16 nonequivalent octahedral iron positions of varying valence (between 2.32 and 2.95) established by the bond valence sum (BVS) method. The majority of $\mathrm{Fe} B$ atoms are grouped into characteristic cigarlike three-atom structures, dubbed trimerons $[25,26]$, with the central atom of type-2+ valence (actually, 2.32-2.55) and outer ones more of 3+ valence (between 2.36 and 2.95). Despite the uncertainties in determining the valence state by the BVS method in a highly covalent bonding, this complex structure suggests the presence of nearly disordered arrangements prone to easy changes under external conditions and fluctuations close to the disordering temperature $T_{V}$.

The trimeron structure was in accord with local probes such as NMR [27]. However, although all the lines from the $24 \mathrm{Fe}$ positions in ${ }^{57} \mathrm{Fe}$ NMR spectra are very well separated (see also Refs. [28,29]), this technique is not directly valence sensitive and information about the electric field gradients is not accessible in the case of the spin- $1 / 2{ }^{57} \mathrm{Fe}$ nuclei. Consequently, a unique mechanism of the atomic rearrangements below and at the Verwey transition had not yet been discovered. The support from other microscopic techniques, such as Mössbauer spectroscopy, may help to clarify it; this is what we have undertaken here. The 24 different Fe atomic positions should result in the same number of sextets in a Mössbauer spectroscopy experiment, yielding spectra too complex for reliable analysis. However, Řezníček et al. [30] have shown that these 24 sextets naturally break into 
four groups: eight $\mathrm{Fe} A$ positions, eight less anisotropic $\mathrm{Fe} B$ positions, five more anisotropic $\mathrm{Fe} B$ positions, and three $\mathrm{Fe} B$ sites with anisotropy comparable to the previous group yet with special orientation of the principal axes of the hyperfine field anisotropy tensor. Differences in parameters defining the sextets [hyperfine magnetic fields $B_{\text {eff }}$, electric field gradient affecting quadrupole splitting (QS), and isomer shift (IS)] within these groups are too subtle to be revealed separately and the differences among these groups are relatively high. Consequently, only four groups were proposed to be important for the analysis, and this procedure was successfully applied in the interpretation of Mössbauer spectra in stoichiometric [30] and Zn-doped magnetite [11]. The same procedure will be used here, although it is strictly valid only in a zero external magnetic field [29], as discussed below.

\section{B. Easy axis switching}

Since magnetite structure changes from high-temperature cubic to monoclinic $C c$, each of the six cubic $\langle 100\rangle$ directions may become the low-temperature monoclinic $c_{M}$ axis. As a result, the material breaks into 24 structural domains (twins) unless an external magnetic field $B>0.2 \mathrm{~T}$ is applied along a particular $\langle 100\rangle$ direction while cooling through the transition. This particular direction will then determine both the unique $c_{M}$ axis and the magnetically easy axis. As the $c_{M}$ axis may still be slightly tilted towards four directions (as mentioned above, the monoclinic $\beta$ angle is approximately $0.2^{\circ}$ ), eight crystallographic twins are still present. ${ }^{1}$ If now the sample is magnetized along another (cubic) $\langle 100\rangle$ direction below $T_{V}$ (and higher than $50 \mathrm{~K}$ ), a reorientation of the magnetic moments may take place and this $\langle 100\rangle$ direction becomes a new easy axis $[15,16]$, as schematically presented in Fig. 3. This is known in the literature as axis switching.

We have performed extensive studies of the AS phenomenon by magnetism-oriented experiments [17-20] and by resistivity experiments [31]. It was demonstrated [18] that not only the magnetic but also the crystallographic $c_{M}$ axis is forced in this particular direction. Therefore, the manipulation of the structure by twin domain wall motion is possible by the magnetic field. Importantly, NMR studies have shown that magnetization changes in the field were linked with the microscopic reorganization, thus confirming that these two effects have the same origin [21]. Finally, we confirmed previous Calhoun results that the activation energy $U$ of the AS process, approximately $350 \mathrm{~K}$, is of the same order of magnitude as the Verwey transition temperature [18]. One might thus expect that the magnetic field can trigger the same electronic processes that disorder does at $T_{V}$. In other words, we may expect that all the ordering parameters, including atomic rearrangements, charge, and orbital orderings may be altered by a magnetic field. Thus, understanding how the AS proceeds should help to elucidate the details of the Verwey transition mechanism.

\footnotetext{
${ }^{1}$ They may be avoided by the simultaneous application of field cooling and the uniaxial pressure application; this procedure was not attempted here.
}

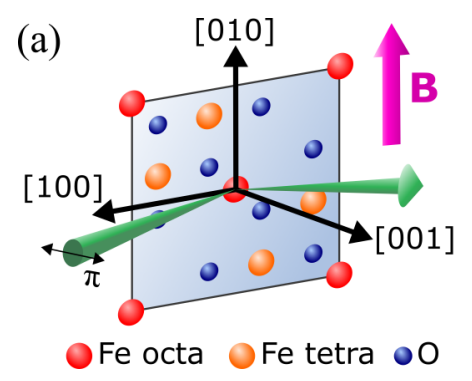

(b)

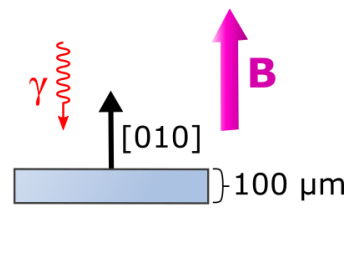

FIG. 1. (a) RXS experimental setup. The flat sample, with [100] and [001] directions in the horizontal scattering plane, is exposed to the vertical magnetic field, i.e., along the [010] direction. The green arrows indicate the incident and scattered beam directions. (b) MS experimental setup. The ${ }^{57}$ Co source was placed above the sample, in the vertical configuration. The $\gamma$ rays penetrate the sample along the [010] direction, along the magnetic field.

\section{EXPERIMENT}

Single crystals of magnetite were prepared by the skull melting method [32] at Purdue University and annealed in $\mathrm{CO} / \mathrm{CO}_{2}$ atmospheres in order to achieve stoichiometry $[33,34]$. The quality of the samples was demonstrated by the very sharp Verwey transition observed by AC magnetic susceptibility (shown in Fig. 1S in [35]). Sample 1 with $T_{V}=123.7 \mathrm{~K}$ was used in the RXS experiments and sample 2 with $T_{V}=122.7 \mathrm{~K}$ was used for Mössbauer spectroscopy measurements. The small difference in $T_{V}$ is likely due to a slight nonstoichiometry of the latter. Based on the universal curve [20], we expect the nonstoichiometry parameter $3 \delta$ in $\mathrm{Fe}_{3(1-\delta)} \mathrm{O}_{4}$ of the order of $7 \times 10^{-4}$ for sample 2. Despite the small variation in the stoichiometry, no significant differences in the electronic properties were observed between the two samples. Furthermore, we measured the influence of the magnetic field on the Mössbauer spectrum at $T>T_{V}$ in a nonannealed, slightly nonstoichiometric single crystal (sample 3), with $T_{V}=120.8 \mathrm{~K}$. Despite the lower $T_{V}$ in this crystal, the trimeron structure was not altered much in comparison with the stoichiometric sample 2 [36] and we can safely assume that the results away from the Verwey transition are representative of pure magnetite. Mössbauer spectra at $160 \mathrm{~K}$ without the external magnetic field of all measured samples are compared in Fig. 1S in [35]. Samples of similar high quality were used in a number of studies on single-crystalline magnetite samples [37-40].

All the crystals were cut with the surface parallel to $\{100\}$ planes (we will use cubic notation) (Fig. 1). In both experiments, we compared the results of three protocols: (1) after the sample was field cooled (FC) with the magnetic field aligned along the cubic axis [010] (i.e., only the twins with the $c_{M}$ axis along the cubic [010] direction formed), (2) after the sample was cooled without a magnetic field (ZFC) (all possible twins formed, i.e., the $c_{M}$ axes pointed along [100], [010], and [001]), and (3) after the sample was ZFC and a magnetic field was subsequently applied along [010] (i.e., the direction from the first arrangement) at $T<T_{V}$. We would expect similar results from the first and last arrangements. The details will be outlined below, separately for each experiment. 


\section{A. Resonant $x$-ray scattering}

Resonant X-ray scattering experiments were carried out at the former ID20 beamline of the ESRF in Grenoble, France [41]. Sample 1, a flat plate with an area of approximately $4 \times$ $5 \mathrm{~mm}^{2}$ and $1.5 \mathrm{~mm}$ thick, was placed in an Oxford Instrument split 10-T superconducting magnet. The (001)-plane vector of the sample was approximately horizontal with a vertical [010] axis along the magnetic field direction. The cryostat was placed on a four-axis goniometer. A Si(111) doublecrystal monochromator was used to select the x rays (energy resolution of $0.8 \mathrm{eV}$ at these photon energies) of the required energy near the Fe $K$ edge $(E=7.119 \mathrm{keV})$. The incident beam was linearly polarized in the horizontal plane, i.e., also in the scattering plane ( $\pi-\pi$ polarization). The scattered beam polarization was analyzed by means of a $\operatorname{MgO}(222)$ analyzer crystal in both $\sigma$ and $\pi$ channels. A sample geometry with respect to the incident beam is shown in Fig. 1(a).

Sample resistance is sensitive to AS [20,31] and thus it was monitored in situ throughout the experiment. A standard four-point resistance measurement technique was used with the RS830 lock-in amplifier employed as the current source and for reading the voltage on the sample.

\section{B. Mössbauer spectroscopy}

The samples used for MS measurements were first glued to a $0.1-\mathrm{mm}$ sapphire plate and then polished down to $0.1 \mathrm{~mm}$ thickness. Neither the glue nor the sapphire plate showed any significant MS absorption. The measurements of ${ }^{57} \mathrm{Fe}$ Mössbauer spectra were performed in the transmission geometry using a constant acceleration type of spectrometer in a vertical Janis cryostat equipped with a 9-T superconducting magnet. Further experiments at zero magnetic field were performed in an ICE Oxford cryostat. The ${ }^{57} \mathrm{Co}$ in $\mathrm{Rh}$ source was placed in the cryostat above the sample and was shielded from the magnetic field. Both spectrometers were calibrated at room temperature with a $10-\mu$ m-thick $\alpha$-Fe foil. The sample was placed with the [010] direction parallel to the $\gamma$ ray and magnetic field [Fig. 1(b)]. Due to the source at low temperature, IS in acquired spectra is offset by $-0.25 \mathrm{~mm} / \mathrm{s}$ with respect to values referencing $\alpha$-Fe at room temperature.

The spectra were analyzed by means of the least-squaresfitting procedure, which entailed calculations of the positions and relative intensities of the absorption lines by numerical diagonalization of the full hyperfine interaction Hamiltonian $[42,43]$. The absorption line shape of the spectra was described using a transmission integral formula $[44,45]$. A more detailed description of the fitting procedure is provided in Ref. [35] [see Fig. 3S(a) in [35] for the coordination system used]. Four components were fitted at $T<T_{V}$, as in Refs. [11,30] and two components at $T>T_{V}$.

\section{RESULTS}

\section{A. Resonant x-ray scattering}

Electronic transitions from fully occupied electronic orbitals to empty or nearly empty orbitals located near the Fermi level can be photon induced. The process is characterized by an absorption edge at a given energy and its position depends on the oxidation state of the resonant atom. It is the signature of both the geometrical and electronic local environment around it and the long-range order related to the (magnetic) space group. Thus Fe ions with different charge states are expected to have their absorption edges at slightly different energies. In the case of iron, the transition $1 s \rightarrow 4 p$ yields the $K$ edge at about $7.119 \mathrm{keV}$ and it has been used to monitor charge and orbital orderings [7,22]. In contrast, the $L$ edges $(2 p \rightarrow 3 d)$ are located at much lower energies and experiments at these edges have been used to monitor orbital ordering as well as changes in the magnetic ordering $[6,46]$.

When recorded at weak or forbidden reflections, RXS can be used to measure the charge ordering, that is, the charge difference between atoms, which could have been equivalent before a phase transition. This sensitivity comes from the phase factor $P_{j}=\exp \left(-i \pi \boldsymbol{r}_{j} \cdot \boldsymbol{Q}\right)$ and the positions $\boldsymbol{r}_{j}$ of the pair of atoms projected along the $\boldsymbol{Q}$ direction. For specific reflections, they are close to being opposite, and the resulting structure factor $F(\boldsymbol{Q}, E)=\left[A\left(f_{1}+f_{2}\right)+B\left(f_{1}-f_{2}\right)+F_{0}\right]$, with $A=1 / 2\left(P_{1}+P_{2}\right)$ and $B=1 / 2\left(P_{1}-P_{2}\right)$, nearly measures the difference between the two scattering factors $f_{1}$ and $f_{2}$ of the two atoms which could be, for example, $\mathrm{Fe}^{2+}$ and $\mathrm{Fe}^{3+}$. Here $F_{0}$ is the contribution of the other atoms of the unit cell. Thus, the sensitivity of RXS to electronic charge order (CO) is maximized for reflections where the $A$ parameter is relatively small, or ideally zero. Since they correspond to different charge states, their respective absorption edges will have a slightly shifted position $f_{1}(\omega) \sim f_{2}(\omega+\delta)$. The difference stresses this fact and a peak is expected at the resonance [22]. Note that here $f_{1}$ and $f_{2}$ are scalars. The same kind of equation can be formulated for orbital ordering (OO) except in this case $f_{1}$ and $f_{2}$ will be tensors or components of a tensor once the incoming and outgoing photon beam polarizations are taken into account [47].

In order to find the optimal conditions (e.g., photon energy) to observe a particular electronic order, $\mathrm{CO}$ or $\mathrm{OO}$, we first calculated the energy dependence of selected peaks, which may be sensitive to these phenomena and which we aimed to observe during the AS process. The calculations, performed within the FDMNES package [48], used the procedure discussed in detail in Refs. [7,49] and the results are presented in Fig. 2. In the case of OO, we relied on the calculations shown in Ref. [49]. Following Refs. [25,26], the atomic positions were imbedded with the trimeron structure; charge and orbital orders were simplified after [24]. The following reflections were selected as significant to the problem (the cubic unit cell is used to label the Bragg peaks).

(a) The (004) peak is visible in the low- and the high$T$ phases, measured off-resonance, at $E=7.06 \mathrm{keV}$. The changes in the lattice symmetry at $T_{V}$ are marked by a step in the intensity vs temperature relation (for details see Fig. $2 \mathrm{~S}$ in [35]). The relative intensities of the (400), (040), and (004) peaks are nearly the same and the intensity is weakly dependent on energy.

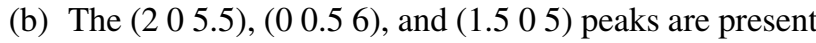
only in the low- $T$ phase, due to crystal lattice doubling in the $c_{M}$ direction, measured off-resonance.

(c) The (003) peak is present only in the low- $T$ phase, measured off-resonance, at $E=7.06 \mathrm{keV}$, where the intensity is larger than at resonance, in the $\pi-\pi$ polarization channel. This peak is not sensitive to electronic orders. The intensity of 


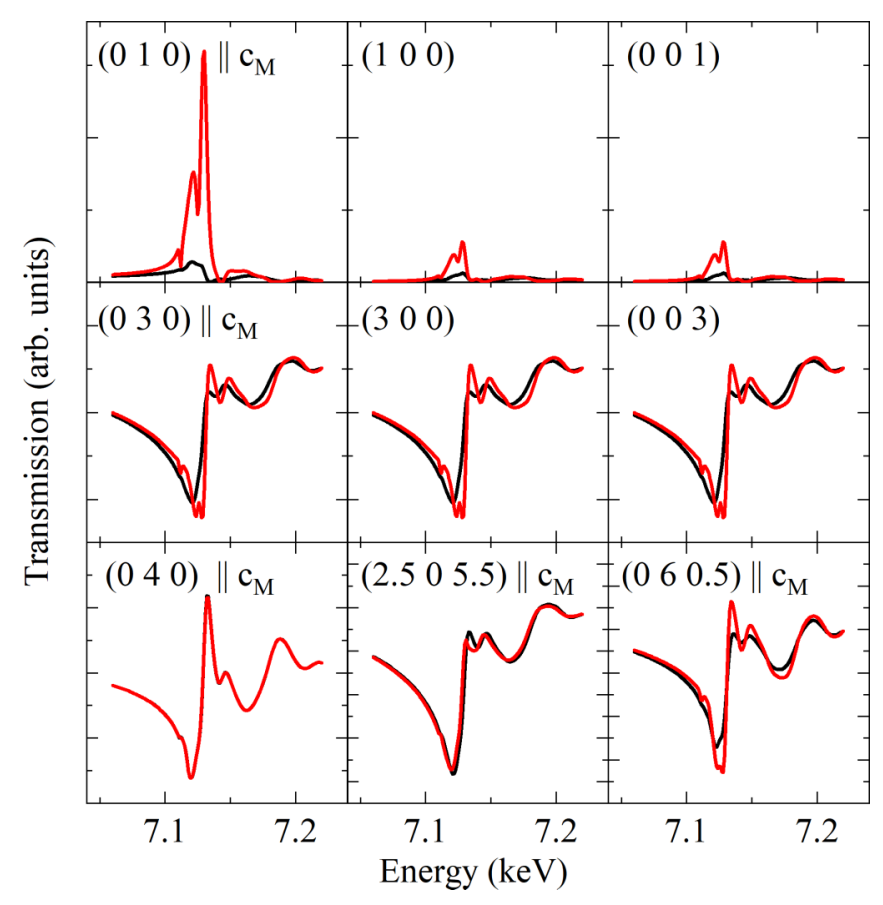

FIG. 2. FDMNES calculation of selected reflections within the $C c$ space group, assuming the absence (black curves) and presence (red curves) of charge order. For (040), all other reflections [(400) and (004)] are identical and no indication of charge order may be drawn.

the (030) peak is twice that of (300) and (003), so the relative changes related to AS are expected. The energy dependence of these three reflections is nearly identical.

(d) The (001) peak is present only in the low- $T$ phase, measured at a resonant energy of $E=7.119 \mathrm{keV}$, where it has a particularly large amplitude due to the charge order. The (100) [or (010) and (001)] reflections are sensitive to different charge configurations (see Fig. 2 and [7]). The former measures the difference between $\mathrm{Fe}(2.5+)+\mathrm{Fe}(3+)$ and $\mathrm{Fe}(2+)$, whereas the latter is the difference between $\mathrm{Fe}(2.5+)$ $+\mathrm{Fe}(2+)$ and $\mathrm{Fe}(3+)$. The charge ordering at $(010)$ can be seen as the difference between the contributions at $B 1$ sites and $B 2$ sites (here sites are labeled according to Ref. [24]); the $B 3$ and $B 4$ sites hardly contribute to the $\mathrm{CO}$ at this reflection.

(e) The $\left(\begin{array}{lll}0 & 0 & 5.5\end{array}\right)$ and $\left(\begin{array}{lll}0 & 0 & 3.5\end{array}\right)$ peaks are present only in the low- $T$ phase and are extinct in classical diffraction, observed in the $\pi-\sigma$ polarization channel at resonance, at $E=7.119 \mathrm{keV}$; these reflections are related to the orbital order $[7,46,47,50]$.

The temperature dependence of selected reflections marking the occurrence of the Verwey transition is presented in Fig. $2 \mathrm{~S}$ in [35]. Clearly, the transition is sharp and well reflected both in the (004) peak (allowed in both $F d \overline{3} m$ and $C c$ symmetries) and in those extinct in the high- $T$ phase, (003) and (001), although there is an apparent difference between (004) and (003) from one side and (001) from the other.

Selected reflections were measured in each of the three sets of experiments, according to the protocol introduced in Sec. II and schematically shown in Fig. 3(a). ${ }^{2}$ First, the sample was $\mathrm{FC}$ down to $8 \mathrm{~K}$ in the magnetic field of $2 \mathrm{~T}$, high enough to uniquely define the basic $c_{M}$ axis along the [010] direction [51] [left panel in Fig. 3(a)].

In the following experiment, the sample was $\mathrm{ZFC}$ to $8 \mathrm{~K}$, resulting in 24 crystallographic domains, with $c_{M}$ axes vertical ([010] direction), horizontal in the plane of the sample (direction [100]) or horizontal, normal to the sample surface ([001] direction), as shown in the middle panel of Fig. 3(a). In both cases the cubic direction is actually monoclinic $a_{M}-b_{M}$ diagonal, an unspecified magnetic direction. These three sets of twins appear to have approximately a similar volume; however, possible strain caused by the sample mounting may influence the relative twin distribution. After ZFC, followed by the diffraction measurements, the sample was then warmed up to $63 \mathrm{~K}$ and, in a separate experiment, to $110 \mathrm{~K}$. In order to induce the AS, magnetic fields of 2 and $1.1 \mathrm{~T}$ were applied at the two temperatures, respectively [Fig 3(a) right panel].

The results presented in Fig. 3 (first four graphs of the left column) demonstrate that field cooling the sample aligns the $c_{M}$ axis along [010] (along the magnetic field), resulting in a single basic domain seen by the intense $(00.56)$ peak and the absence of (1.5 05 ), (2 0 5.5), and (0 05.5$)$, all as expected. Consequently, ZFC results in the multitwin structure reflected

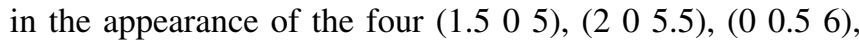
and $\left(\begin{array}{lll}0 & 0 & 5.5\end{array}\right)$ peaks, which are furthermore insensitive to the external magnetic field applied at low $T=8 \mathrm{~K}$ (shown in Fig. 6S in [35] for clarity), again according to our expectations. The twins are particularly well seen in the double structure of the $\left(\begin{array}{lll}2 & 0 & 5.5\end{array}\right)$ peak. The $\left(\begin{array}{lll}0 & 0 & 1\end{array}\right)$ and $\left(\begin{array}{lll}0 & 0 & 3\end{array}\right)$ peaks were expected to exist after both FC and ZFC, although after ZFC their intensities should increase rather than decrease, as the experiment showed (see also Fig. 2).

After the magnetic field was applied at $63 \mathrm{~K}$ and the AS occurred, the intensities of the studied peaks changed: The

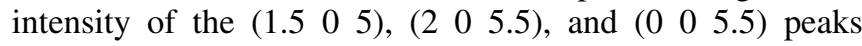
decreased significantly, as expected in the case where the magnetic field switches the domains with $c_{M}$ along [100] and [001] into a single domain with $c_{M}$ along the [010] axis. Surprisingly, and contrary to our expectations, the intensity of $\left(\begin{array}{lll}0 & 0.5 & 6\end{array}\right)$ did not increase after AS. With the decrease of the (l.5 05 5) and ( $\left.\begin{array}{lll}2 & 0 & 5.5\end{array}\right)$ peaks one would expect the spectral weight transfer to the (0 0.56$)$ peak as an effect of a single-domain formation with $c_{M}$ aligned along [010] and the intensity of this reflection to be comparable to the case of cooling in the magnetic field along the [010] axis.

After the magnetic field was switched off, the peaks did not return to their initial shape, demonstrating that the ASinduced reorganization of the structure is stable. A similar situation, although not so clear, occurs when the AS is attempted at $110 \mathrm{~K}$ (shown in Fig. 7S in [35]), where bulk magnetization

\footnotetext{
${ }^{2}$ Here the cubic structure is presented in a schematic way to show only the magnetic field direction versus monoclinic $c_{M}$. The important fact that in case $B$ is perpendicular to $c_{M}$, it is along the $a_{M}-b_{M}$ diagonal is not pointed out, but this will play a role in the MS experiment discussion.
} 


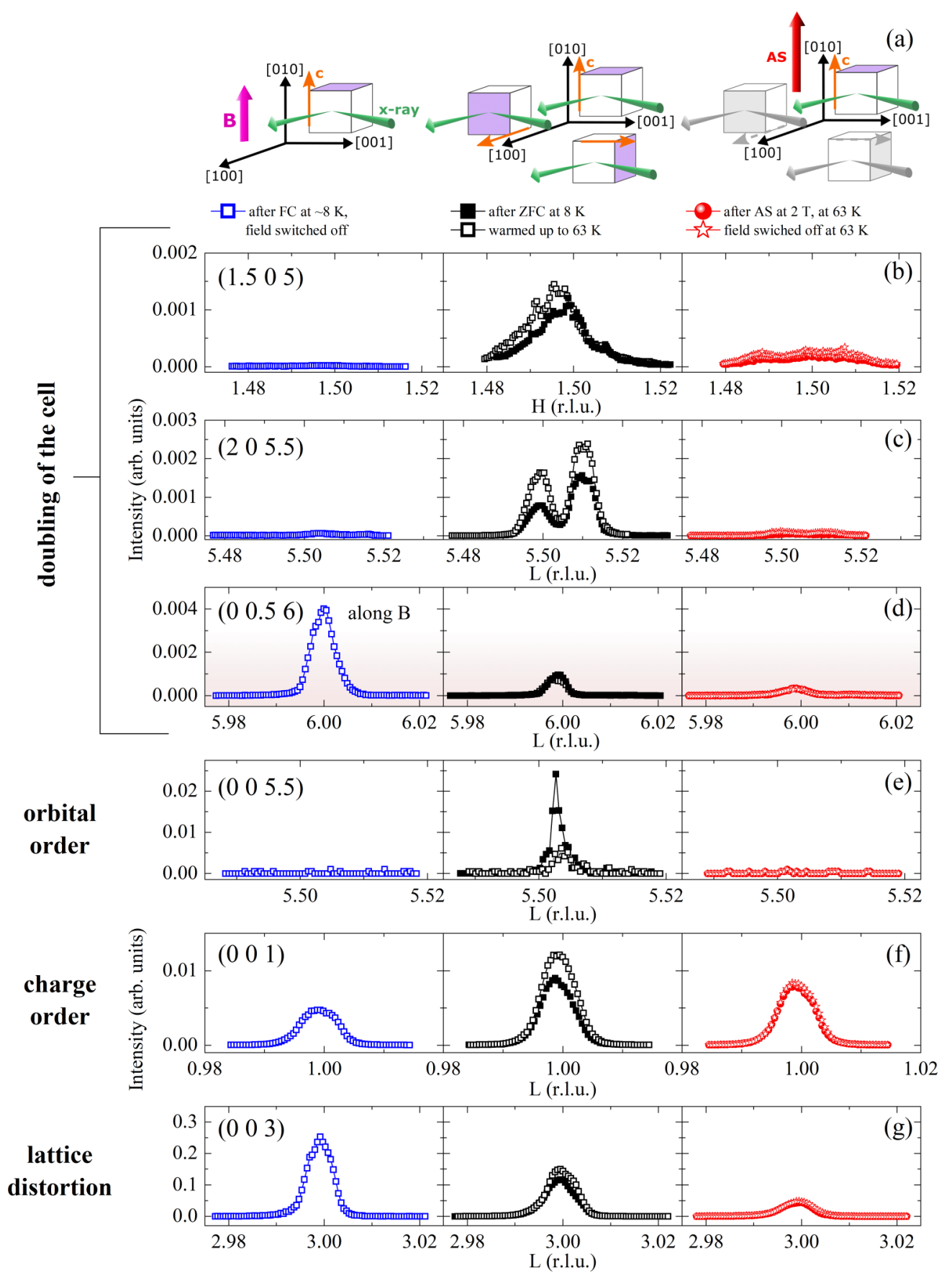

FIG. 3. Summary of the RXS experiment. (a) Schematics of the experimental protocol. See the text for details. (b)-(g) Momentum scans of the representative Bragg peaks sensitive to the structural transition and electronic orders as indicated. The pink shaded panel corresponds to the $\left(\begin{array}{ll}0 & 0.56)\end{array}\right)$ peak representing the doubling of the unit cell along the direction of the external magnetic field. The data collected after FC are presented in the left column, after ZFC in the middle column, and after AS under a magnetic field of $2 \mathrm{~T}$ at $63 \mathrm{~K}$ is shown in the right column. In the case of ZFC (middle column) the data were collected at a base temperature $(\sim 8 \mathrm{~K})$ as well as at the temperature at which AS was performed. The proof that the structure does not change under a magnetic field at the lowest temperature is presented in Fig. 6S in [35]. The peaks were measured off-resonance $(E=7.05 \mathrm{keV})$, except (001) and ( 005.5$)$, as described in the text.

experiments show a stable axis switching [18]. The response of the (003), (001), and ( 00.56$)$ peaks to this experimental procedure is more complex and the reasons for this behavior will be discussed in Sec. IV A. This last result suggests that much more stringent conditions have to be implemented in the experimental protocol, and they were applied to the Mössbauer experiment presented in the next section.

\section{B. Mössbauer spectroscopy}

Based on the results shown above and with the aim to observe how axis switching affected magnetic and electronic states of monoclinic magnetite, the following layout of the experiment, shown schematically in Fig. 4, was realized for sample 2. 

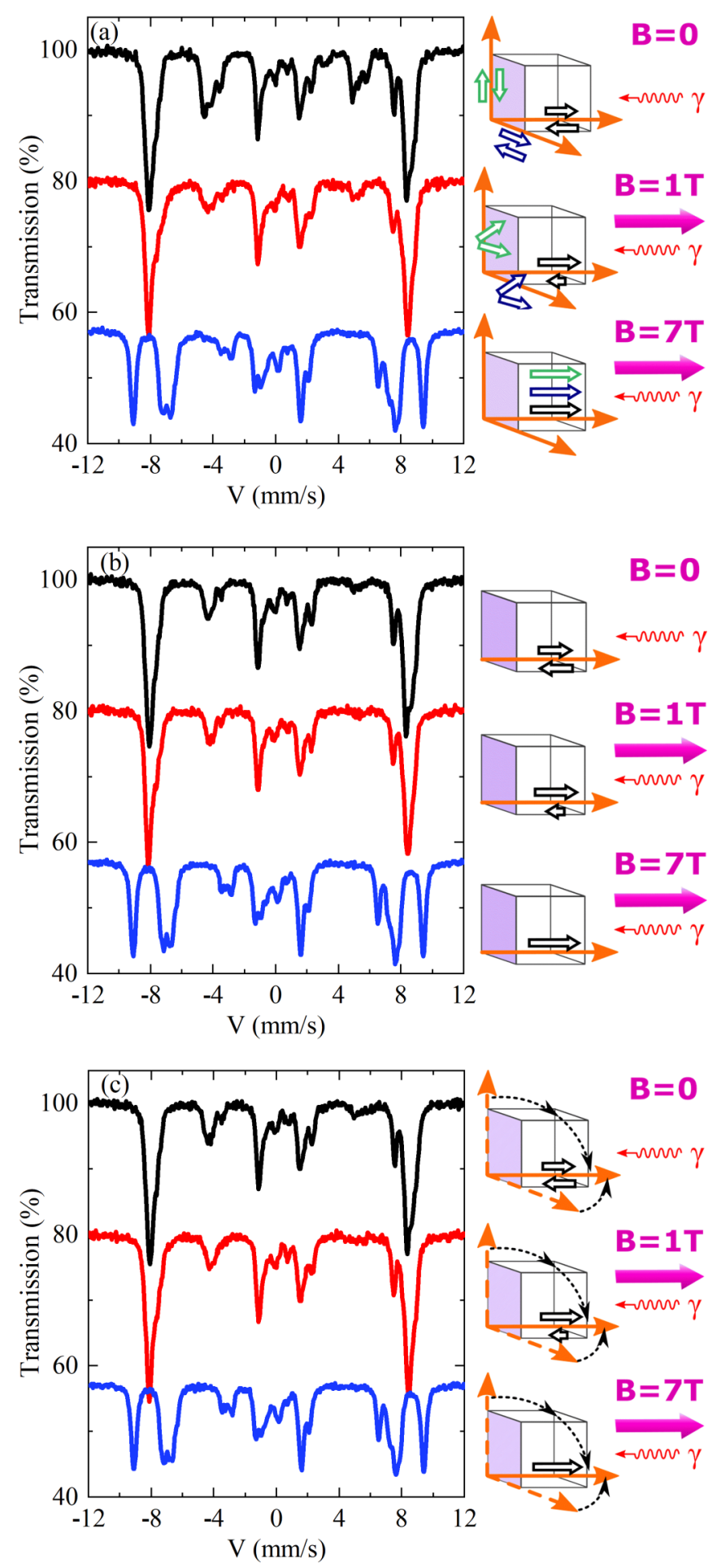

FIG. 4. MS spectra at $4.2 \mathrm{~K}$ (each component is shifted to allow for better separation; the scale for each component is identical). The schematics of crystallographic $c_{M}$ axes (thin orange arrows) and main magnetic domains (hollow arrows) presented on the top of the high- $T$ cubic cell show how the $c_{M}$ axes are expected to change in different magnetic fields (bold magenta arrows). The wavy line shows the $\gamma$ ray direction. (a) MS spectra after ZFC, (b) MS spectra after FC, (c) MS spectra after the sample was ZFC to $4.2 \mathrm{~K}$, heated to $90 \mathrm{~K}$, followed by 7-T field application along the [010] axis, and finally measured at $4.2 \mathrm{~K}$. (i) The sample was first cooled from room temperature (RT) to $4.2 \mathrm{~K}$ without a magnetic field, resulting in a multidomain state both crystallographically and magnetically. Basically, three main directions of $c_{M}$ axes should be present [marked by three orange arrows in Fig. 4(a)], together with a complex magnetic domain structure (since $c_{M}$ are easy magnetic axes, the main domains have their magnetization vectors pointing in these three directions, shown as hollow arrows in Fig. 4). A Mössbauer experiment was then performed at $B=0,1$, and $7 \mathrm{~T}$ vertical magnetic field along [010] [i.e., perpendicular to the (010) sample surface; as before, in Fig. 3, the important fact that once $B$ is perpendicular to $c_{M}$ it is along the monoclinic $a_{M}-b_{M}$ diagonal is not shown, but plays a role in the discussion].

(ii) In the next set of experiments [Fig. 4(b)] the sample was FC $(B=4 \mathrm{~T})$ from RT to $4.2 \mathrm{~K}$ and subsequently measured in the same fields $B=0,1$, and $7 \mathrm{~T}$ as in the previous experiment. This time, however, right after cooling the sample should basically have one $c_{M}$ axis, which should also limit the number and shape of magnetic domains, i.e., the magnetic domain structure is different from that in case (i). Thus, one of the aims of our experiments might be to see how sensitive MS is to the apparently different magnetic domain landscape in view of the totally different crystal domain structure.

(iii) In the AS experiment, the sample was $\mathrm{ZFC}$ down to $4.2 \mathrm{~K}$, as in (i), which again resulted in a multidomain structure with the main $c_{M}$ axes pointing along all the $\langle 100\rangle$ directions. The sample was then heated to $90 \mathrm{~K}$ and a magnetic field of $7 \mathrm{~T}$ was applied for $20 \mathrm{~min}$ along the plane normal. The goal of our experiment was to check that only crystallographic domains with their $c_{M}$ axes close to the plane normal should now be present. After the magnetic field was applied at $90 \mathrm{~K}$, the sample was again cooled to $4.2 \mathrm{~K}$ and measured in the same set of fields $B=0,1$, and $7 \mathrm{~T}$ as before. The results, together with the symbolic experiment layout, are presented in Fig. 4(c).

Since the main aim of the experiment was to see if the spectra after FC were similar to those after AS was made, in Fig. 5 we show the difference in spectra after ZFC and after FC (red circles) and the difference of the spectra after AS and after FC (black line). If our suggestion is supported by the experimental results, those last spectra should be close to zero.

Additionally, in a process of sample preparation, we have collected MS spectra at a few temperatures in the vicinity of the Verwey transition in the other setup (ICE Oxford, without a magnetic field) [see Fig. 3S(b) in [35]]. We have also measured another, slightly nonstoichiometric single crystal, sample 3, at $160 \mathrm{~K}$ and in fields $0,4,7$, and $9 \mathrm{~T}$. The results are presented in Fig. 4S(a) in [35].

\section{DISCUSSION}

Many measurements made on stoichiometric magnetite single crystals, focused on various properties and performed with different techniques, proved that a sufficiently large magnetic field applied at temperatures right below $T_{V}$ causes AS. Thus, our aim was to see how the changes in different physical properties triggered by AS are reflected in the results from the symmetry probe (RXS) and local probe (MS). 


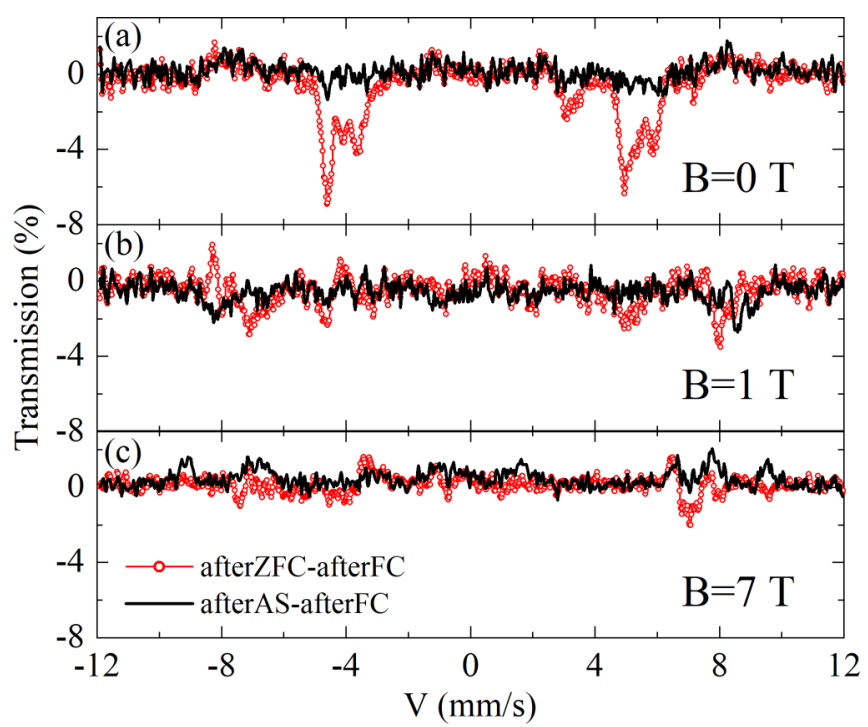

FIG. 5. Difference in MS spectra at $4.2 \mathrm{~K}$ after ZFC and FC (red circles) and after AS and FC (black line) measured (a) in the zero magnetic field, (b) at $1 \mathrm{~T}$, and (c) at $7 \mathrm{~T}$.

In general, the results show that the axis switching occurs and both techniques can be used to observe this phenomenon. However, this conclusion is much better supported by MS results and RXS data are much more ambiguous. We will discuss the results separately for each experimental technique.

\section{A. Resonant $x$-ray scattering}

The aim of the RXS measurements was to understand the microscopic mechanism of AS by observing the changes of different subsystems, charge ordering, crystal lattice distortion, and orbital ordering, while AS takes place. Our results, shown in Fig. 3 and in Fig. 7S in [35], compare the process of inducing a single domain by cooling the sample across $T_{V}$ in the magnetic field along the [010] direction with aligning the three domains along this direction using the magnetic field in the AS process. Here we discuss the response of each subsystem to the applied procedure.

The first observation is that cooling across $T_{V}$ in the magnetic field seems to induce solely domains with $c_{M}$ along the [010] direction. Although direct proof would require the emergence of peaks, e.g., ( $\left.\begin{array}{lll}0 & 1.5 & 5\end{array}\right)$ and (2 5.50$)$, which should be created by FC, they could not be observed due to limitations of the geometry. At the same time, the peak with

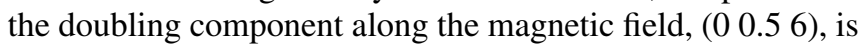
very intense.

In the next sequence, we tested if a magnetic field applied along [010] can remove the domains with $c_{M}$ along [100] and [001] created after ZFC or, in other words, if AS is observed (right column in Fig. 3). Applying the magnetic field at a base temperature of $\sim 5 \mathrm{~K}$ does not influence the diffraction peaks (shown in Fig. 6S in [35]), but after heating up to $\sim 65 \mathrm{~K}$ and field application, the (1.5 05 ) and (2 05.5$)$ peaks almost entirely vanished, as after FC, signaling the occurrence of AS, according to the expectations. However, the area of the $\left(\begin{array}{lll}0 & 0.5 & 6\end{array}\right)$ peak did not increase to the value observed after FC, but, on the contrary, its intensity slightly decreased.
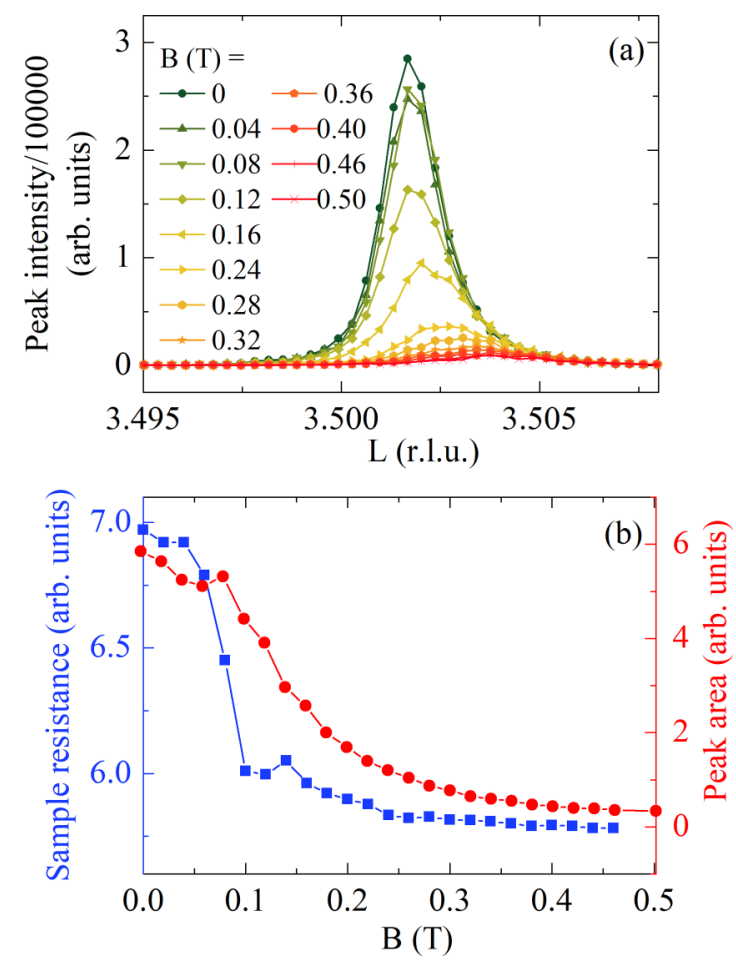

FIG. 6. Magnetic field dependence of (a) the shape and (b) the area of $\left(\begin{array}{lll}0 & 0 & 3.5\end{array}\right)$ reflection measured in resonance at $110 \mathrm{~K}$ in $B=0.02 \mathrm{~T}$ steps [only selected spectra are shown in (a)]. The peak area is correlated with sample resistance in (b), clearly showing the axis switching process starting under $B=0.1 \mathrm{~T}$. The left, blue axis describes sample resistance, while the right, red axis describes the peak area.

Removing the magnetic field after AS occurred does not influence significantly the peak intensity, demonstrating that the magnetic field forms a stable structure at approximately $65 \mathrm{~K}$.

The OO is revealed by ( $00 n / 2)$-type peaks only visible in the $C c$ structure at resonance. Our experiment shows that the ( 000 5.5) reflection is sensitive to AS: The intensity of the peak vanishes entirely after the process of AS and is equivalent to cooling in the magnetic field [Fig. 3(c)], as expected. This demonstrates that one of the basic domains, with $c_{M}$ along [001], i.e., 1/3 of the sample, undergoes a complete rearrangement. Due to the experimental setup limitations, the $\left(\begin{array}{lll}5.5 & 0 & 0\end{array}\right)$ or $(05.50)$ reflections were not accessible in the scattering plane. Therefore, the OO peak of the domain with $c_{M}$ along the magnetic field direction, signaling a quasi-onedomain structure after AS, could not be confirmed.

Also the other $O O$ peak, (l $\left.\begin{array}{lll}0 & 0 & 3.5\end{array}\right)$ observed at $110 \mathrm{~K}$, behaves in a similar way, confirming a change of orbital ordering triggered by the magnetic field. The intensity of this reflection vs increasing magnetic field at $110 \mathrm{~K}$ is shown in Fig. 6 and is correlated with sample resistance that also changes once AS occurs $[31,20]$. However, the peak almost totally recovers after magnetic field release (Fig. 7S in [35]), which might indicate that the orbital order is not stable, contrary to what

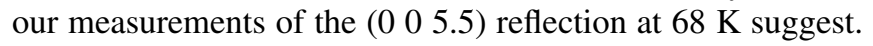
As shown in Refs. [6,52], the crystallographic structure in the proximity of the VT (i.e., at $110 \mathrm{~K}$ ) is not stable and 
continuous rearrangements of the domains occur. Thus, we cannot claim that the AS concerns only lattice distortion and not orbital order.

The other reflection that shows the electronic processes triggered at the Verwey transition is (010). Our calculations (see Fig. 2) and also the results in Ref. [7] show that this peak is sensitive to the charge disproportionation within octahedral sites once the photon energy is tuned at the Fe $K$ edge. Also, this sensitivity is much more pronounced for (010), in the case in which the cubic lattice is doubled along the [010] direction, than (001). For experimental geometry reasons, the (010) peak could not be observed here and only the changes of (001) are reported.

After ZFC the twins with $c_{M}$ along [001] appeared, so the intensity of (001) increased in contrast to the situation after FC [left and middle panels in Fig. 3(d), respectively]. Although the behavior of this peak at $8 \mathrm{~K}$ after FC and its increased intensity after ZFC agree with the calculations, we expected the intensity of this peak to decrease with field application both at $63 \mathrm{~K}$ [Fig. 3(d)] and at $110 \mathrm{~K}$ (Fig. 7S in [35]). Instead, the peak did not react to the application of the field. A similar situation was observed for the (003) peak (measured off-resonance; this is not sensitive to the $\mathrm{CO}$ as shown in Fig. 2) that is more pronounced after FC than after ZFC. Although the peak weakened after AS, as expected based on the calculations in Fig. 2, the whole sequence cannot be easily rationalized based on the simple meaning of AS.

The temperature dependence of the charge ordering (001) peak measured at resonance is different from the lattice distortion peaks (003) and (004). These last two peaks change at $T_{V}$ abruptly, unlike (001), which is present up to $130 \mathrm{~K}$ and has a characteristic increase at $T_{V}$ (see Fig. $2 \mathrm{~S}$ in [35]). This behavior at $T_{V}$ confirms the results in Ref. [7] that the processes responsible for it survive $10 \mathrm{~K}$ above $T_{V}$ whereas lattice distortion, measured by (003) and (004), already disappeared. Since the measured reflections display lattice symmetry, whether observed off-resonance or at resonance, the difference in the respective transition temperature suggests that those symmetries are different. As already pointed out in Ref. [9], local and more global atomic arrangements behave differently with temperature. While the former, pertaining to orbital and charge order and therefore somehow linked to the trimeron fluctuations, survive up to the Curie temperature, the longer range global fluctuations only subsist up to $100 \mathrm{~K}$ above $T_{V}$ (and those still more global to $T_{V}$ ). Here we postulate that the puzzling reaction to the application of a magnetic field is linked to the different nature of the measured reflections, more global [such as (004)] or more local [such as (001)]; one of these might be rather inactive at $T_{V}$ and under trimeron manipulation by the magnetic field.

The possible alternative approach to the unexpected changes in some peaks may concern surface effects and the complicated near-surface structural domains that appear in magnetite structure in the temperature region close to the Verwey transition $[52,53]$. The near-surface structure is additionally affected by strain developed during sample polishing prior to the experiment [54]. Consequently, it is not excluded that it may behave differently from that in the bulk and also reacting to the external factors, such as magnetic field, in a different way. The x-ray attenuation length above but close to resonance, $7.119 \mathrm{keV}$, is $6.5 \mu \mathrm{m}$ and differs from that below resonance energy $E=7.07 \mathrm{keV}, 46 \mu \mathrm{m}$. At $E=14.4 \mathrm{keV}$, as in MS measurements, the attenuation length is $42 \mu \mathrm{m}$. It is then not excluded that near-surface related effects may be observed at (l 003.5$)$, ( $\left.\begin{array}{lll}0 & 0 & 5.5\end{array}\right)$, and (001) (see also [55]) as well. Further, since thermal relaxation occurs faster in the surface region than in the bulk, which is additionally amplified by the relatively high temperature, $110 \mathrm{~K}$, this relaxation is quicker than at lower $T$, resulting in the mainly surface related behavior.

It was recently suggested that collective processes such as phase transitions and also axis switching as considered here require the combined action of electronic and various structural deformations, both static and dynamic, on a scale beyond a few lattice constants [56]. Also, our modeling of AS based on purely structural arguments (not presented here) shows that some disorder after AS is unavoidable. All these features suggest the possibility of long-range structural inhomogeneities and thus hints at an alternative way to explain the intriguing facts presented above.

In any case, the experience with RXS has led us to learn and correct our approach to better target the MS experiments. Namely, all MS measurements were performed at $4.2 \mathrm{~K}$ and larger magnetic fields in the FC procedure $(4 \mathrm{~T})$ and in switching the axis (7 T) were applied.

\section{B. Mössbauer spectroscopy}

A simple inspection of the MS results (Figs. 4 and 5) leads to important conclusions. As expected, MS spectra after AS are very similar to those obtained after FC in $4 \mathrm{~T}$. From the MS results we can confirm our predictions that the magnetic field causes such an electronic rearrangement and atomic displacement that the structure after AS, at least to the accuracy of MS, is very similar to that obtained after FC. The fitting procedure, parameters, and their comparison are discussed below.

We have fitted MS spectra following the method described in Ref. [30] and based on the grouping of all 24 different Fe positions, $8 A$ and $16 B$, into one and three components, respectively (see [35]). This is done also in the magnetic field, although, in the case of the ZFC treatment, the grouping is justified only in a zero external field, as shown below. To make the discussion easier, the spectra of the sample treated in three different ways (ZFC, FC, and ZFC followed by magnetic field application) and separately for each field are presented in Fig. 7.

\section{Zero-field spectra}

In a similar way as in Ref. [30], we initially assumed that for each magnetic domain within a twin domain the magnetization vector $M$ points along the local $c_{M}$ axis. Twins with $c_{M}$ in plane (forming roughly $2 / 3$ of a sample) thus give rise to a $3: 4: 1: 1: 4: 3$ (lines are counted from the left, of lowest energy, number 1, to the right, number 6; see Fig. 8, where the simulation of line intensity is presented) intensity ratio of the sextet lines [see Fig. 8(a)] and those with $c_{M}$ pointing along the $\gamma$-ray direction to a 3:0:1:1:0:3 ratio [see Fig. 8(b)]. Since, after zero-field cooling, those first twins should be twice more abundant than the latter, the net result would be close to that in 

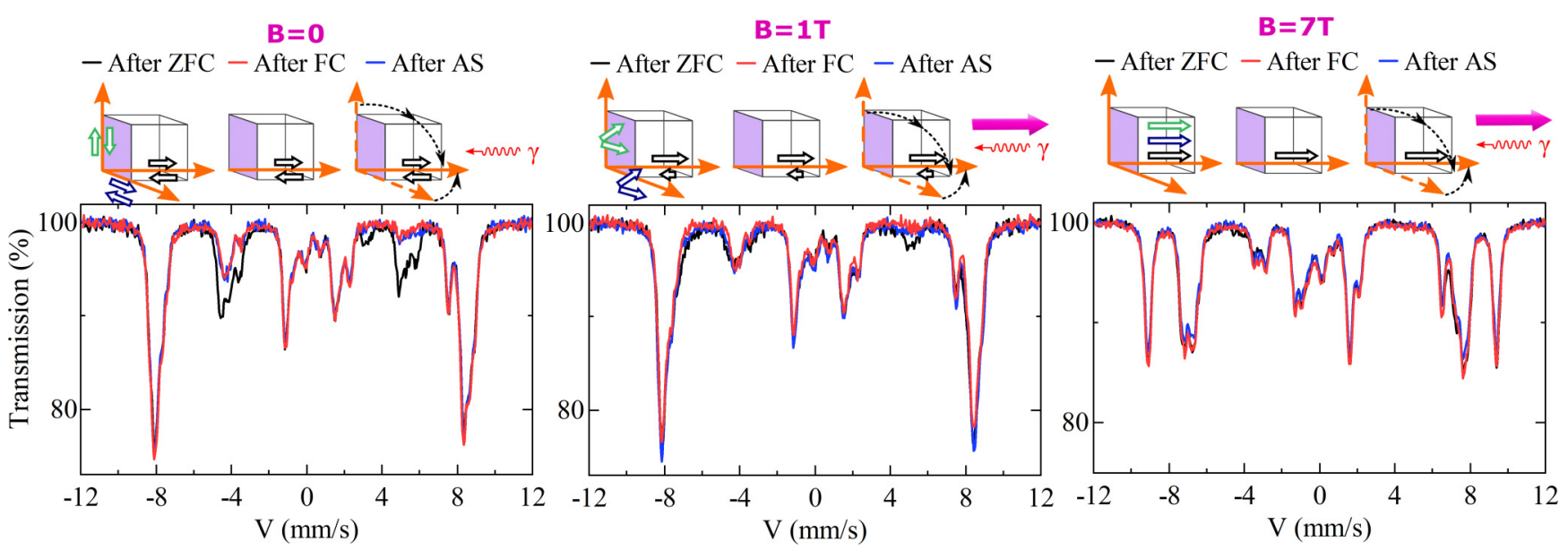

FIG. 7. MS spectra of the stoichiometric magnetite single crystal, treated in different ways, under various external magnetic fields. Note that although the samples after FC and after AS occurred are very similar (identical, within error bars), the one after ZFC, where twins with $c_{M}$ in several directions are present, is different.

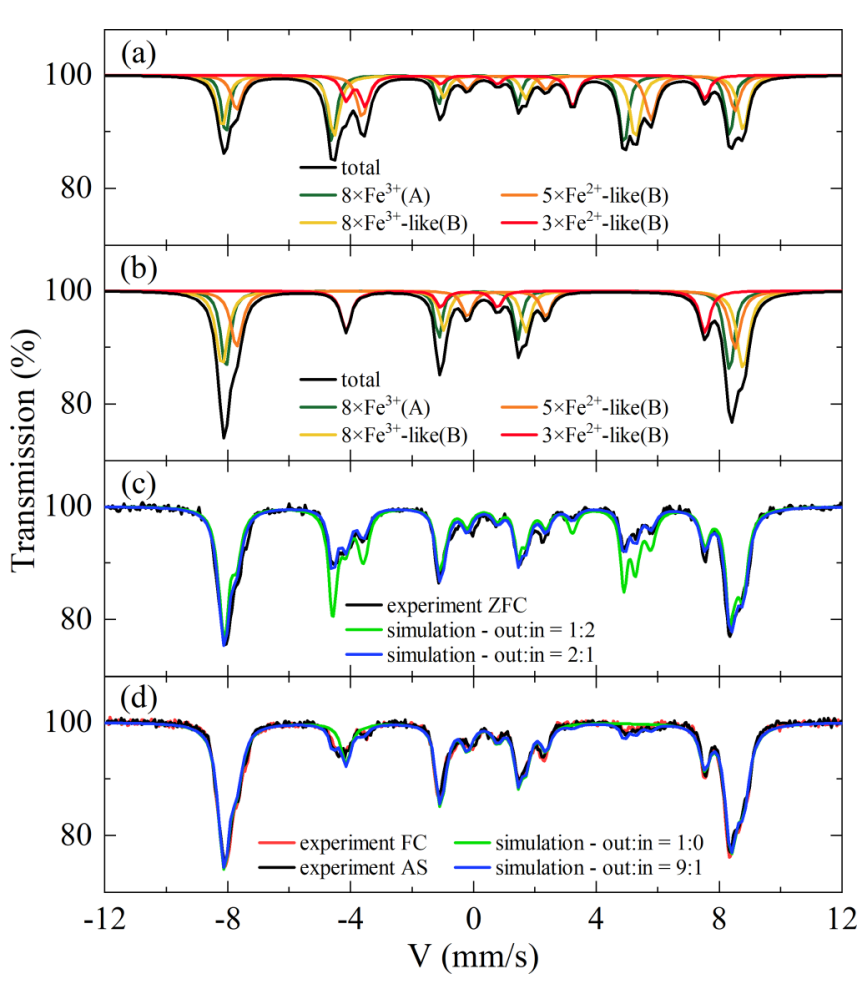

FIG. 8. Simulated and experimental zero-field MS spectra of the stoichiometric magnetite single crystal under consideration of various ratios of domains with in-plane and out-of-plane magnetization direction (denoted by out:in): (a) simulation for in-plane magnetization direction, (b) simulation for out-of-plane magnetization, (c) spectra after ZFC, and (d) spectra after FC and AS. The simulations (carried out using the FITSUITE 1.1.1.RC.10 software [57]) are based on the four-component model from [30] and hyperfine parameters from Table VI in Ref. [30] [line broadening of the $\mathrm{Fe}^{3+}$ (-like) and $\mathrm{Fe}^{2+}$-like components was reduced by $60 \%$ and $30 \%$, respectively, to match this experiment better]. For the sake of simplicity, demagnetizing effects were neglected in the simulations. a powder sample. However, the spectral pattern observed here indicates a higher abundance of domains with out-of-plane magnetization than expected. Although flux closure domains with $M$ along directions other than $c_{M}$ may exist, neither this strain nor a strain possibly resulting from gluing the sample to sapphire plate seems to suffice to explain the observation. Taking into account the small remanence of magnetite, the spin-reorientation transition, and the fundamental changes of domain structure during cooling from $300 \mathrm{~K}$ to $4 \mathrm{~K}$, even possible nonzero initial bulk magnetization of the sample would unlikely be the dominant cause. We repeated the measurement (including ZFC from temperature well above $T_{V}$ ) and we obtained essentially the same spectrum. Therefore, we believe that the observed line intensities are probably due to a configuration of defects which may locally affect preferred $c_{M}$ orientation. The defect configuration is unique for every sample, even for almost stoichiometric single crystals such as those investigated here. The ideal situation, as well as a more realistic one closer to the experimental data, is illustrated by simulations in Fig. 8(c).

The situation is different after the sample was either FC or if AS occurred: All $c_{M}$ axes should point along the cubic [010] direction and the number of magnetic moments along the $\gamma$-ray direction should increase accordingly. This means that the spectral components with an intensity ratio of 3:0:1:1:0:3 should prevail. This is indeed the case as shown in Fig. 7(a); the absorption structure between 2.8 and $6.3 \mathrm{~mm} / \mathrm{s}$ (corresponding to line 5 of each of the four components) is almost completely missing after FC and AS (except for a very small peak at $4.95 \mathrm{~mm} / \mathrm{s}$ ). The same effect should be observed for line 2 (between -5.5 and $-2.7 \mathrm{~mm} / \mathrm{s}$ ). Here, however, the absorption structure is lowered, but still observed. The visible absorption is to some extent due to an overlap with line 1 of the low-field $3 x \mathrm{Fe}^{2+}$-like $(B)$ component. The experimental data are compared with simulations considering the ideal case as well as a situation better matching the experimental data in Fig. 8(d). Note that a tilt of magnetization in domains along the edges of the sample might be a more likely scenario than the simulated pessimistic estimate of $10 \%$ of the sample volume containing domains with $c_{M}$ (and $M$ ) in the plane. 
To conclude, the differences between the spectra of the sample after ZFC and after FC or AS are linked with changes of relative sextet line intensities of the four spectral components. Thus, the abundance of domains with out-of-plane and in-plane directions of magnetization and $c_{M}$ can be estimated. Despite the nonideal initial ratio of the domains with outof-plane and in-plane magnetization in the initial ZFC case, the corresponding minimization of the number of domains with in-plane magnetization both after FC and AS is clearly evidenced. Moreover, the spectra of the sample after FC and after AS are essentially the same, which indicates complete AS in the latter case.

\section{High-field spectra}

In the presence of a magnetic field high enough to completely magnetize the sample $[7 \mathrm{~T}$ in these studies; see Fig. 7(c)], all magnetic moments should point along the magnetic field direction and simultaneously along the $\gamma$-ray direction. ${ }^{3}$ However, the twin structure after the sample was either FC or AS occurred is completely different from that after ZFC: In $1 / 3$ of the sample $c_{M}$ axes point along [010], the field direction, while $c_{M}$ axes are perpendicular to the field in the remaining $2 / 3$ of the sample. The hyperfine field $\mathrm{Fe}$ nucleus experienced in the presence of a 1.3-T magnetic field along various directions was measured by Mizoguchi [29] and was later simulated by the density-functional-theory (DFT) analysis by Řezníček et al. [27]. The results are summarized in Fig. 2 in Ref. [29] and Fig. 1 of [27]; the data say that, in general, the hyperfine fields at $\mathrm{Fe} B$ nuclei are different from when the magnetic field is along $c_{M}$, which may hinder the grouping of $16 B$ spectra into three well-resolved groups. In particular, $80 \%$ of the first $B$ component [composed of the spectra from the eight least anisotropic $B$ positions $(C 2$ in Fig. 5S in [35])] is close to that with $c_{M}$ along the magnetic field, with the remaining $20 \%$ adding to the background. The second component, composed of the spectra of five more anisotropic $B$ sites (C3 in Fig. 5S in [35]), should again be restored to $70 \%$, the rest again spread due to other hyperfine fields. The largest changes should occur for the third $B$ component [composed of the spectra from the three most anisotropic sites ( $C 4$ in Fig. 5S in [35])], where the change in hyperfine field is approximately $15 \mathrm{~T}$. This component is expected to split into $33 \%$ with a small field of $36 \mathrm{~T}$ and $66 \%$ with a field of approximately $44 \mathrm{~T}$. The details of hyperfine field estimation for each $\mathrm{Fe} B$ position are discussed in Ref. [35].

Besides the hyperfine field anisotropy, also the impact of the electric field gradient on Mössbauer spectra, the quadrupole splitting, changes when magnetization is not along $c_{M}$. This was already encountered and addressed in Ref. [30], but unlike for $B_{\text {eff }}$, no experimental data exist. Again, we

\footnotetext{
${ }^{3}$ Actually, despite a high field, the magnetization vector in the domains with the $c_{M}$ axes in-plane may not be completely realigned with the external field, since the crystallographic direction along the field is approximately $45^{\circ}$ off either the hard ( $a_{M}$ axis) or intermediate $\left(b_{M}\right)$ magnetic direction. We expect that the departure from full magnetization is negligible at $7 \mathrm{~T}$ and small at $1 \mathrm{~T}$ and does not hinder the discussion below.
}

expect that the QS does not change much for $A$ sites and, to a lesser certainty, in less anisotropic $B$ sites (forming the eight $B$ site $C 2$ component); however, the other two components may be affected more.

Summarizing, the four-component model that was suggested in Ref. [30], was successfully used in Ref. [11], and is used here may not be applicable when the magnetization is rotated to some direction other than along $c_{M}$. Consequently, we might expect that the MS spectrum in the magnetic field (especially as large as $7 \mathrm{~T}$ ) after ZFC will be substantially different from the relevant spectra after FC and AS.

Surprisingly, our MS results show that all spectra, after $\mathrm{ZFC}, \mathrm{FC}$, and $\mathrm{AS}$, at $7 \mathrm{~T}$ are identical within the error bar [Fig. 7(c)]. The only difference that could be spotted is in the energy region between -4.7 and $-3.8 \mathrm{~mm} / \mathrm{s}$, where very small absorption in the ZFC sample can be seen. Apparently, MS is not so sensitive to the direction of magnetization with respect to crystallographic axes, or the changes of spectra are hidden in those lines, 2 and 5, that were extinct when incoming quantum momentum is along magnetization.

\section{Intermediate-field spectra}

For the intermediate 1-T field, qualitatively similar problems with $B_{\text {eff }}$ and QS changing with magnetization directions are encountered. Additionally, the field of $1 \mathrm{~T}$ is not strong enough to fully turn the magnetization into the direction of the external field: The magnetization vector avoided the $a_{M}-b_{M}$ plane inclining toward the $c_{M}$ axis, even up to $20^{\circ}$ [27]. This means that lines 2 and 5 may not be completely extinct. Further, Fig. 2 from [29] suggests that the anisotropic behavior in the $a_{M}-c_{M}$ plane as well as in the $b_{M}-c_{M}$ plane is quite pronounced. Therefore, an even larger part of the signal from the ZFC case at zero magnetic field may be smeared/broadened at the 1-T field after ZFC.

Again, in some contrast to our expectations, our 1-T spectra of the sample after ZFC, FC, and AS are very similar, except in the energy region $4.48-5.73 \mathrm{~mm} / \mathrm{s}$, where the kink, i.e., some higher absorption after ZFC, is still observed (there are also some other very small yet visible differences around $-7 \mathrm{~mm} / \mathrm{s})$. This is either due to different $B_{\text {eff }}$ and QS in a large part of the sample (caused by the magnetic field aligned in a direction other than $c_{M}$, as explained above) or because at the 1-T field not all moments are along the $\gamma$-ray quantum direction and lines 2 and 5 are not completely extinct.

In the first scenario, which is less probable than the other, if the kink at approximately $5 \mathrm{~mm} / \mathrm{s}$ is caused by the sixth line of some component, the $B_{\text {eff }}$ of this component should be approximately $32 \mathrm{~T}$ and this value could only be close to that obtained from the $C 3$ component [i.e., the $\mathrm{Fe} B 2+-$ like component (see Fig. 5S in [35] and Fig. 2 of [29])], which suggests a $B_{\text {eff }}$ of approximately $41 \mathrm{~T}$ for the $B 5$ and $B 10$ lines). In the second, much more probable, scenario, the kink is due to a not-completely-extinct line 5 of the same C3 component. One branch of the split signals from the $\mathrm{Fe}$ sites constituting this component does not change much in comparison to the case with $B$ along $c_{M}$ (Fe sites $B 5, B 10$, $B 12, B 7$, and $B 13$, Fig. 2 in Ref. [29]), i.e., it should be at almost the same position as at $B=0$. 
An alternative explanation to the unexpected result that all (ZFC, FC, and after AS) MS spectra at $1 \mathrm{~T}$ are very similar may be that spin and orbital moments are decoupled, as found recently at room temperature [58]. When the field is applied, the spin moments readily point along the field direction, while small spin orbit coupling and orbital magnetic moments lead to a two-step process: At low fields the small orbital moment, at least from certain $B$ positions, still remains in plane and thus gives rise to some absorption (the kink at approximately $5 \mathrm{~mm} / \mathrm{s}$ ). At higher fields this remaining orbital moment also aligns along the external field and essentially no difference between ZFC and FC (and after AS) is seen. Orbital order manipulation with field was also observed by RXS [peaks ( 0 0 5.5) and ( $\left.\begin{array}{lll}0 & 0 & 3.5\end{array}\right)$ discussed above].

The results of Mössbauer spectra analysis, with only $B_{\text {eff }}$, $V_{z z}$, and IS fitted, are shown in Ref. [35] in Fig. 5S and in Tables $1 \mathrm{~S}(160 \mathrm{~K})$ and $2 \mathrm{~S}-4 \mathrm{~S}(T=4.2 \mathrm{~K})$. The external field dependence of $B_{\text {eff }}$, giving the demagnetization field $B_{D}$, is presented and discussed in Figs. 4S(b) and 4S(c) in Ref. [35] for the high-temperature and low-temperature phases, respectively. The general conclusion is that hyperfine parameters are very similar for sample 2 treated in different ways. It shows that the pattern of $\mathrm{Fe}$ atom charges (affecting IS) and the orbital ordering (affecting the electric field gradient $V_{z z}$ ) are the same before and after AS, but the atoms that have this pattern are different. This constitutes the main result of our studies.

Axis switching is realized in the insulating phase of magnetite and is connected with $B$ atom orbitals reorganization. This is a low- $T$ realization of the scenario at high $T$ shown in Ref. [59], where trigonally split states originating from lowest $t_{2 g}$ orbitals dynamical change in the bond lengths related to the $X 3$ phonon mode (responsible for the Verwey transition [59]) and Kugel-Khomskii interactions, all of similar energy below $100 \mathrm{meV}$, play a role in the magnetic field dependence of the magnetic moment. Although this mechanism was found at $T>T_{V}$, all the important ingredients of it have their origin at the low- $T$ structure. At low $T$ only lower energy levels are occupied, but both an increase of temperature or some other external trigger (magnetic field in our case) may cause some excited levels to be admixed. When temperature is increased, trimeron dynamics [9], anharmonic effects, [60] and short-range order revealed in x-ray diffuse scattering [8] replace static low- $T$ phenomena. A similar mechanism involving the same interactions was described in Ref. [61]. Also, the vibrations of the trimerons' centers ( $\mathrm{Fe} B 2+$ ions) are of lower energy than the surrounding $\mathrm{Fe}$ atoms [62], which additionally confirms a pronounced dynamics within $\mathrm{Fe}$ cluster surrounding the $\mathrm{Fe} B 2+$ atom.

We therefore suspect that fluctuations within six nearest neighbors of the trimeron centers' $\mathrm{Fe} B 2+$ atoms are large at $T<T_{V}$ and thus their combined electronic state may be very fragile and susceptible to any external trigger such as magnetic field. This can cause electronic rearrangement, i.e., trimeron order change, despite the fact that a large energy, of the order of $1 \mathrm{eV}$, is needed to rotate an individual trimeron [62]. Only the combined effect of several static (e.g., trigonal distortion) and dynamic (phonons, especially of $X 3$ symmetry) correlations, extending beyond a few lattice cells, may cooperate and diminish the energy barrier separating different
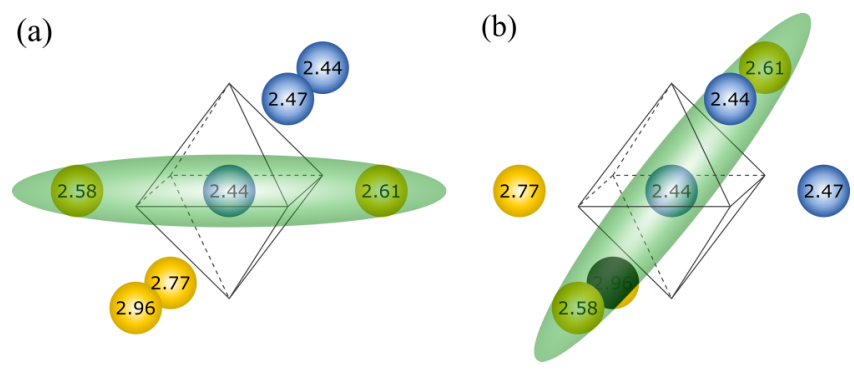

FIG. 9. (a) $\mathrm{Fe} B$ atom that does not change its symmetry (i.e., also its valence and the valence of nearest neighbors) in AS. The color differentiates $\mathrm{Fe} B$ valence (those with valence beyond 2.5 are yellow) and the trimeron is marked with the cigar shape. Monoclinic $c_{M}$ is vertical. The numbers denote $B$ atom valences as shown in Ref. [25,26] (b) Same atom and its surroundings after AS; a new $c_{M}$ is now horizontal. Note that although the central Fe cation is still the trimeron center, the trimeron direction has changed.

ionic arrangements [56]. Thus, once the temperature is closer to $T_{V}$ (e.g., 68 and $110 \mathrm{~K}$ in our case of RXS and $90 \mathrm{~K}$ in MS) the energy-activated hopping between those $\mathrm{Fe} B$ orbitals that are parts of trimerons sets in. This process leads to a strongly correlated, nonlocal, but still energy-activated action: the combined change of trigonal component of the crystal field that lowers the $\mathrm{Fe} B$ orbital energy in the magnetic field and the surrounding oxygen atoms' slight adjustments (extending beyond a few lattice constants and still lowering the energy). All these lead to the electron transfer between orbitals and the ensuing change of trimeron order, both observed as the axis switching. This mechanism concerns additional (to the one described recently in Ref. [62]) trimeron arrangement excitation.

The experimentally obtained activation energy of this process (approximately $350 \mathrm{~K}$ [17-20], close to the excitations found in Ref. [61]) should be compared to the difference in energy between the states with a magnetic field along $c_{M}$ and perpendicular to $c_{M}$ (the diagonal of $a_{M}-b_{M}$ ) that can be calculated from first principles. It should be noted that this activation energy increases under any small disorder in the sample (as caused by low-level $\mathrm{Zn}$ doping or nonstoichiometry) [20].

There are a few $\mathrm{Fe} B 2+$ sites (like that shown in Fig. 9) that do not change their symmetry in AS: Those $\mathrm{Fe} B$ cations transfer in AS to the same symmetry position but with reoriented surrounding. Thus, both the valence of this particular $\mathrm{Fe} B 2+$ atom and the total valence of its nearest-neighbor $\mathrm{Fe} B$ atoms are not changed. These sites may inhibit a long-distance electronic transfer initiated by activation processes: Magnetite is not a conductor in the process of axis switching [although AS is signaled by the resistance change [31], as also shown in Fig. 6(b)], but the electronic exchange between orbitals is a precursor of such a long-distance electronic transfer. In contrast, at the Verwey transition, i.e., at few tens of $\mathrm{K}$ above the temperature of our AS observation, all channels, including those that do not change in AS are activated: The initial activation process leads to a self-activated exchange of charge between all $\mathrm{Fe} B$ atoms seen as an insulator-semiconductor Verwey transition. This correlated transfer averages out local symmetry to globally observed cubic, although this symmetry 
is unstable [11] and fluctuations in the form of trimerons persist up to room temperature $[8,60]$ or even up to $T_{C}$ [9].

Thus, trimeron collective change at AS is a precursor to the Verwey transition and a probable model of electronic fluctuations above VT. Therefore, we believe that further observation of the axis switching and the attempt to construct its microscopic mechanism will shed light on trimeron formation and structure, their excitations leading to the Verwey transition, and how these electronic fluctuations cooperate/cause a ferrimagnetic to paramagnetic transition (as suggested in Ref. [9]).

\section{CONCLUSION}

We have presented experimental results showing that the axis switching phenomenon in the magnetite single crystal can be observed by a local probe (MS) and a probe sensitive to the sample symmetry (RXS). In particular, we observed that all subsystems ordered below the Verwey transition temperature, i.e., lattice distortion, charge, and orbital ordering unanimously react to the magnetic field.

Our MS results, fitted and analyzed according to the method based on DFT calculations and published recently [30,11], fully confirm the occurrence of AS: The field-cooled sample with basically one $c_{M}$ axis has the same spectrum as the one obtained after ZFC followed by magnetic field application at $90 \mathrm{~K}$.

Similar results were obtained from RXS: Some peaks, previously calculated by FDMNES, signaling lattice distortion, charge, and orbital orderings, present after ZFC, changed or decreased in a way similar to the FC sample after a magnetic field was applied and the AS occurred. In a few cases, the results were odd, e.g., the peak regained its value prior to field application or the peak did not change in a predictable way; the situation is still under debate but suggests the important role of the surface effects in the interpretation of x-raydiffraction results.

Although discerning a valence change of individual ions during AS was not possible, orbital reorganization after an external magnetic field is applied and after this application triggers the axis switching certainly occurs. We also suggested that although $\mathrm{Fe} B$ atom orbital reorganization observed in AS precedes the Verwey transition, higher temperature is needed to trigger fluctuations within all $\mathrm{Fe} B$ atomic orbitals and between them, which results in a smearing of the $C c$ symmetry, i.e., the Verwey transition. Thus, a microscopic description of this precursor and its observation, possibly with MS and/or XRD and/or neutron diffraction as a function of time, linked with existing NMR results, can shed more light on the mechanism of AS, the Verwey transition, as well as on trimeron fluctuations at still higher temperatures.

\section{ACKNOWLEDGMENTS}

This work was partially supported by the Faculty of Physics and Applied Computer Science AGH UST from a subsidy of the Ministry of Science and Higher Education of Poland. I.B. and W.T. were supported by the Polish National Agency for Academic Exchange under "Polish Returns 2019” Program No. PPN/PPO/2019/1/00014/U/0001. Access to ESRF was financed by the Polish Ministry of Science and High Education through Grant No. DIR/WK/2016/19.
[1] K. Renger, Die anfangliche suszeptibilitat von eisen und magnetit in abhangigkeit von der temperatur, Ph.D. thesis, Eidgenossischen Technischen Hochschule Zürich, 1913.

[2] S. Sahling, J. E. Lorenzo, G. Remenyi, C. Marin, V. L. Katkov, and V. A. Osipov, Heat capacity signature of frustrated trimerons in magnetite, Sci. Rep. 10, 10909 (2020).

[3] M. Bałanda, A. Wiecheć, D. Kim, Z. Kąkol, A. Kozłowski, P. Niedziela, J. Sabol, Z. Tarnawski, and J. M. Honig, Magnetic AC susceptibility of stoichiometric and low zinc doped magnetite single crystals, Eur. Phys. J. B 43, 201 (2005).

[4] Z. Švindrych, Z. Janů, A. Kozłowski, and J. M. Honig, Lowtemperature magnetic anomaly in magnetite, Phys. Rev. B 86, 214406 (2012).

[5] K. Yamauchi, T. Fukushima, and S. Picozzi, Ferroelectricity in multiferroic magnetite $\mathrm{Fe}_{3} \mathrm{O}_{4}$ driven by noncentrosymmetric $\mathrm{Fe}^{2+} / \mathrm{Fe}^{3+}$ charge-ordering: First-principles study, Phys. Rev. B 79, 212404 (2009).

[6] R. Kukreja, N. Hua, J. Ruby, A. Barbour, W. Hu, C. Mazzoli, S. Wilkins, E. E. Fullerton, and O. G. Shpyrko, Orbital Domain Dynamics in Magnetite below the Verwey Transition, Phys. Rev. Lett. 121, 177601 (2018).

[7] J. E. Lorenzo, C. Mazzoli, N. Jaouen, C. Detlefs, D. Mannix, S. Grenier, Y. Joly, and C. Marin, Charge and Orbital Correlations at and above the Verwey Phase Transition in Magnetite, Phys. Rev. Lett. 101, 226401 (2008).
[8] A. Bosak, D. Chernyshov, M. Hoesch, P. Piekarz, M. Le Tacon, M. Krisch, A. Kozłowski, A. M. Oleś, and K. Parliński, ShortRange Correlations in Magnetite above the Verwey Temperature, Phys. Rev. X 4, 011040 (2014).

[9] G. Perversi, E. Pachoud, J. Cumby, J. M. Hudspeth, J. P. Wright, S. A. J. Kimber, and J. P. Attfield, Co-emergence of magnetic order and structural fluctuations in magnetite, Nat. Commun. 10, 2857 (2019).

[10] P. Piekarz, K. Parliński, and A. M. Oleś, Mechanism of the Verwey Transition in Magnetite, Phys. Rev. Lett. 97, 156402 (2006).

[11] V. Chlan, J. Żukrowski, A. Bosak, Z. Kąkol, A. Kozłowski, Z. Tarnawski, R. Řezníček, H. Štépánková, P. Novák, I. Biało, and J. M. Honig, Effect of low Zn doping on the Verwey transition in magnetite single crystals: Mössbauer spectroscopy and x-ray diffraction, Phys. Rev. B 98, 125138 (2018).

[12] E. Pachoud, J. Cumby, G. Perversi, J. P. Wright, and J. P. Attfield, Site-selective doping of ordered charge states in magnetite, Nat. Commun. 11, 1671 (2020).

[13] S. Lee, A. Fursina, J. T. Mayo, C. T. Yavuz, V. L. Colvin, R. G. Sumesh Sofin, I. V. Shvets, and D. Natelson, Electrically driven phase transition in magnetite nanostructures, Nat. Mater. 7, 130 (2008).

[14] S. de Jong, R. Kukreja, C. Trabant, N. Pontius, C. F. Chang, T. Kachel, M. Beye, F. Sorgenfrei, C. H. Back, B. Brauer 
et al., Speed limit of the insulator-metal transition in magnetite, Nat. Mater. 12, 882 (2013).

[15] B. A. Calhoun, Magnetic and electric properties of magnetite at low temperatures, Phys. Rev. 94, 1577 (1954).

[16] E. Vittoratos, I. Baranov, and P. P. M. Meincke, Crystal axis switching effects in the thermal expansion and magnetostriction of magnetite, J. Appl. Phys. 42, 1633 (1971).

[17] G. Król, J. Kusz, Z. Tarnawski, Z Kąkol, W. Tabiś, and A. Kozłowski, Studies of the magnetic axis switching in magnetite, Acta Phys. Pol. A 109, 601 (2006).

[18] G. Król, J. Kusz, Z. Tarnawski, Z. Kąkol, A. Kozłowski, and J.M. Honig, Studies of magnetic axis switching phenomenon in magnetite, J. Alloys Compd. 442, 83 (2007).

[19] A. Britwum, T. Kołodziej, W. Tokarz, J. Przewoźnik, C. Kapusta, Z. Kąkol, A. Kozłowski, Z. Tarnawski, and J. M. Honig, Magnetostriction of first and second order magnetite samples and its relation to the magnetic easy axis switching, Solid State Phenom. 194, 120 (2013).

[20] Z. Kąkol, A. Kozłowski, T. Kołodziej, and J. Przewoźnik, Charge rearrangement in magnetite: From magnetic field induced easy axis switching to femtoseconds electronic processes, Philos. Mag. 95, 633 (2015).

[21] V. Chlan, K. Kouřil, H. Štépánková, R. Řezníček, J. Štépánek, W. Tabiś, G. Król, Z. Tarnawski, Z. Kąkol, and A. Kozłowski, Magnetically induced structural reorientation in magnetite studied by nuclear magnetic resonance, J. Appl. Phys. 108, 083914 (2010).

[22] J. E. Lorenzo, Y. Joly, D. Mannix, and S. Grenier, Charge order as seen by resonant (elastic) X-ray scattering, Eur. Phys. J. Spec. Top. 208, 121 (2012).

[23] M. Iizumi, T. F. Koetzle, G. Shirane, S. Chikazumi, M. Matsui, and $\mathrm{S}$. Todo, Structure of magnetite $\left(\mathrm{Fe}_{3} \mathrm{O}_{4}\right)$ below the Verwey transition temperature, Acta Crystallogr. B 38, 2121 (1982).

[24] J. P. Wright, J. P. Attfield, and P. G. Radaelli, Charge ordered structure of magnetite $\mathrm{Fe}_{3} \mathrm{O}_{4}$ below the Verwey transition, Phys. Rev. B 66, 214422 (2002).

[25] M. S. Senn, J. P. Wright, and J. P. Attfield, Charge order and three-site distortions in the Verwey structure of magnetite, Nature (London) 481, 173 (2012).

[26] M. S. Senn, I. Loa, J. P. Wright, and J. P. Attfield, Electronic orders in the Verwey structure of magnetite, Phys. Rev. B 85, 125119 (2012).

[27] R. Řezníček, V. Chlan, H. Štépánková, and P. Novák, Hyperfine field and electronic structure of magnetite below the Verwey transition, Phys. Rev. B 91, 125134 (2015).

[28] P. Novák, H. Štépánková, J. Englich, J. Kohout, and V. A. M. Brabers, NMR in magnetite below and around the Verwey transition, Phys. Rev. B 61, 1256 (2000).

[29] M. Mizoguchi, Charge and orbital ordering structure of $\mathrm{Fe}_{3} \mathrm{O}_{4}$ in the low-temperature phase as deduced from NMR study, J. Phys. Soc. Jpn. 70, 2333 (2001).

[30] R. Řezníček, V. Chlan, H. Štépánková, P. Novák, J. Żukrowski, A. Kozłowski, Z. Kąkol, Z. Tarnawski, and J. M. Honig, Understanding the Mössbauer spectrum of magnetite below the Verwey transition: Ab initio calculations, simulation, and experiment, Phys. Rev. B 96, 195124 (2017).

[31] G. Król, W. Tabiś, J. Przewoźnik, T. Kołodziej, Z. Kąkol, A. Kozłowski, and Z. Tarnawski, Magnetoresistance in magnetite: Switching of the magnetic easy axis, J. Alloys Compd. 480, 128 (2009).
[32] H. R. Harrison and R. Aragón, Skull melter growth of magnetite $\left(\mathrm{Fe}_{3} \mathrm{O}_{4}\right)$, Mater. Res. Bull. 13, 1097 (1978).

[33] R. Aragón, H. R. Harrison, R. H. McCallister, and C. J. Sandberg, Skull melter single crystal growth of magnetite $\left(\mathrm{Fe}_{3} \mathrm{O}_{4}\right)$ - ulvöspinel $\left(\mathrm{Fe}_{2} \mathrm{TiO}_{4}\right)$ solid solution members, J. Cryst. Growth 61, 221 (1983).

[34] H. Flood and D. G. Hill, The redox equilibrium in iron oxide spinels and related systems, Z. Elektrochem 61, 18 (1957).

[35] See Supplemental Material at http://link.aps.org/supplemental/ 10.1103/PhysRevB.102.075126 where additional data and details are shown.

[36] G. Perversi, J. Cumby, E. Pachoud, J. P. Wright, and J. P. Attfield, The Verwey structure of a natural magnetite, Chem. Commun. 52, 4864 (2016).

[37] W. Tabiś, Z. Tarnawski, Z. Kąkol, G. Król, A. Kołodziejczyk, A. Kozłowski, A. Fluerasu, and J.M. Honig, Magnetic and structural studies of magnetite at the Verwey transition, J. Alloys Compd. 442, 203 (2007).

[38] J. Żukrowski, A. Wiecheć, R. Zach, W. Tabiś, Z. Tarnawski, G. Król, N.-T. H. Kim-Ngan, Z. Kąkol, and A. Kozłowski, AC magnetic susceptibility under pressure and Mossbauer effect studies of the isotropy point tip in magnetite, J. Alloys Compd. 442, 219 (2007).

[39] I. Biało, A. Kozłowski, M. Wack, A. Włodek, Ł. Gondek, Z. Kąkol, R. Hochleitner, A. Żywczak, V. Chlan, and S. A Gilder, The influence of strain on the Verwey transition as a function of dopant concentration: Towards a geobarometer for magnetitebearing rocks, Geophys. J. Int. 219, 148 (2019).

[40] T. Kołodziej, A. Kozłowski, P. Piekarz, W. Tabiś, Z. Kąkol, M. Zając, Z. Tarnawski, J. M. Honig, A. M. Oleś, and K. Parliński, Nuclear inelastic scattering studies of lattice dynamics in magnetite with a first- and second-order Verwey transition, Phys. Rev. B 85, 104301 (2012).

[41] L. Paolasini, C. Detlefs, C. Mazzoli, S. Wilkins, P. P. Deen, A. Bombardi, N. Kernavanois, F. de Bergevin, F. Yakhou, J. P. Valade et al., ID20: A beamline for magnetic and resonant $\mathrm{x}$-ray scattering investigations under extreme conditions, J. Synchrotron Radiat. 14, 301 (2007).

[42] N. N. Greenwood and T. C. Gibb, Mössbauer Spectroscopy (Chapman and Hall, London, 1971).

[43] P. Gütlich, E. Bill, and A. X. Trautwein, Mössbauer Spectroscopy and Transition Metal Chemistry: Fundamentals and Applications (Springer, Berlin, 2011).

[44] S. Margulies and J. R. Ehrman, Transmission and line broadening of resonance radiation incident on a resonance absorber, Nucl. Instrum. Methods 12, 131 (1961).

[45] G. K. Shenoy, J. M. Friedt, H. Maletta, and S. L. Ruby, Curve fitting and the transmission integral: Warnings and suggestions, in Mössbauer Effect Methodology, edited by I. J. Gruverman, C. W. Seidel, and D. K. Dieterly (Springer US, Boston, 1974), pp. 277-305.

[46] J. Schlappa, C. Schussler-Langeheine, C. F. Chang, H. Ott, A. Tanaka, Z. Hu, M. W. Haverkort, E. Schierle, E. Weschke, G. Kaindl, and L. H. Tjeng, Direct Observation of $t_{2 g}$ Orbital Ordering in Magnetite, Phys. Rev. Lett. 100, 026406 (2008).

[47] S. R. Bland, B. Detlefs, S. B. Wilkins, T. A. W Beale, C. Mazzoli, Y. Joly, P. D. Hatton, J. E. Lorenzo, and V. A. M. Brabers, Full polarization analysis of resonant superlattice and forbidden x-ray reflections in magnetite, J. Phys.: Condens. Matter 21, 485601 (2009). 
[48] Y. Joly, X-ray absorption near-edge structure calculations beyond the muffin-tin approximation, Phys. Rev. B 63, 125120 (2001).

[49] Y. Joly, J. E. Lorenzo, E. Nazarenko, J.-L. Hodeau, D. Mannix, and C. Marin, Low temperature structure of magnetite studied using resonant x-ray scattering, Phys. Rev. B 78, 134110 (2008).

[50] E. Nazarenko, J. E. Lorenzo, Y. Joly, J. L. Hodeau, D. Mannix, and C. Marin, Resonant X-Ray Diffraction Studies on the Charge Ordering in Magnetite, Phys. Rev. Lett. 97, 056403 (2006).

[51] Z. Kąkol, G. Król, W. Tabiś, T. Kołodziej, A. Wisniewski, H. Štépánková, V. Chlan, J. Kusz, Z. Tarnawski, A. Kozłowski, and J. M. Honig, Easy axis switching in magnetite, J. Phys.: Conf. Ser. 303, 012106 (2011).

[52] W. Tabiś, Structural changes in magnetite in vicinity of the Verwey transition observed with various x-ray diffraction methods, Ph.D. thesis, AGH University of Science and Technology, 2010, available at http://winntbg.bg.agh.edu.pl/rozprawy2/ 10248/full10248.pdf.

[53] W. Tabiś, J. Kusz, N.-T. H. Kim-Ngan, Z. Tarnawski, F. Zontone, Z. Kąkol, and A. Kozłowski, Structural changes at the Verwey transition in $\mathrm{Fe}_{3} \mathrm{O}_{4}$, Radiat. Phys. Chem. 78, S93 (2009).

[54] W. Tabiś, J. E. Lorenzo, A. Kozłowski, T. Kołodziej, Z. Tarnawski, Z. Kąkol, C. Mazzoli, H. C. Walker, N. Jaouen, D. Mannix, C. Marin, and J. M. Honig, Effect of surface polishing and oxidization induced strain on electronic order at the Verwey transition in $\mathrm{Fe}_{3} \mathrm{O}_{4}$, J. Phys.: Condens. Matter 25, 055603 (2013).
[55] J. de la Figuera and J. F. Marco, Magnetite and the Verwey transition, from $\gamma$-rays to low-energy electrons, Hyperfine Interact. 240, 44 (2019).

[56] G. G. Guzman-Verri, R. T. Brierley, and P. B. Littlewood, Cooperative elastic fluctuations provide tuning of the metalinsulator transition, Nature (London) 576, 429 (2019).

[57] S. Sajti, L. Deák, and L. Bottyán, Fitsuite: A general program for simultaneous fitting (and simulation) of experimental data, arXiv:0907.2805.

[58] H. Elnaggar, P. Sainctavit, A. Juhin, S. Lafuerza, F. Wilhelm, A. Rogalev, M.-A. Arrio, C. Brouder, M. van der Linden, Z. Kakol, M. Sikora, M. W. Haverkort, P. Glatzel, and F. M. F. de Groot, Noncollinear Ordering of the Orbital Magnetic Moments in Magnetite, Phys. Rev. Lett. 123, 207201 (2019).

[59] P. Piekarz, K. Parliński, and A. M. Oleś, Origin of the Verwey transition in magnetite: Group theory, electronic structure, and lattice dynamics study, Phys. Rev. B 76, 165124 (2007).

[60] M. Hoesch, P. Piekarz, A. Bosak, M. Le Tacon, M. Krisch, A. Kozłowski, A. M. Oleś, and K. Parliński, Anharmonicity due to Electron Phonon Coupling in Magnetite, Phys. Rev. Lett. 110, 207204 (2013).

[61] H. Y. Huang, Z. Y. Chen, R.-P. Wang, F. M. F. de Groot, W. B. Wu, J. Okamoto, A. Chainani, A. Singh, Z.-Y. Li, J.-S. Zhou et al., Jahn-Teller distortion driven magnetic polarons in magnetite, Nat. Commun. 8, 15929 (2017).

[62] E. Baldini, C. A. Belvin, M. Rodriguez-Vega, I. Ozge Ozel, D. Legut, A. Kozłowski, A. M. Oleś, K. Parliński, P. Piekarz, J. Lorenzana, G. A. Fiete, and N. Gedik, Discovery of the soft electronic modes of the trimeron order in magnetite, Nat. Phys. 16, 541 (2020). 